\title{
Paridades de poder adquisitivo para América Latina y el Caribe, 2005-2013: métodos y resultados
}

\author{
Hernán Epstein y Salvador Marconi
}

RESUMEN

Este trabajo tiene como objetivo presentar algunos aspectos metodológicos y las series del producto interno bruto (PIB) de América Latina y el Caribe con respecto al período 2005-2013, expresadas en paridades del poder adquisitivo (PPA), y señalar algunas limitaciones de este tipo de ejercicio. Se realizan comparaciones con las series (a precios corrientes y constantes) expresadas en dólares con tipos de cambio de mercado, y también con los resultados de la ronda del Programa de Comparación Internacional (PCI) efectuada en 2005. Asimismo, se adelantan algunas hipótesis interpretativas sobre el comportamiento de las principales variables económicas calculadas en el estudio.

PALABRAS CLAVE

CLASIFICACIÓN JEL

AUTORES
Producto interno bruto, poder de compra, precios, análisis comparativo, América Latina

C1, E0, O11

Hernán Epstein es Estadístico Asociado en la División para Asuntos de Tratados de la Oficina de las Naciones Unidas contra la Droga y el Delito. hereps@gmail.com

Salvador Marconi es ex funcionario de la Unidad de Estadísticas Económicas y Ambientales de la Comisión Económica para América Latina y el Caribe (CEPAL). salvadormarconi@hotmail.com 


\section{I}

\section{Introducción}

Disponer de series de paridades del poder adquisitivo (PPA) y de los componentes del producto interno bruto (PIB) —o incluso a niveles más desagregados- es sumamente útil para analizar la competitividad, sustentar procesos de negociación para acuerdos comerciales y adoptar decisiones de política económica. Además, esas series podrían ser utilizadas en las mediciones internacionales de pobreza.

En un reciente trabajo, titulado La pobreza absoluta global cayó casi a la mitad el martes ${ }^{1}$, Dykstra, Kenny y Sandefur (2014) muestran los cambios sustanciales que muchos países experimentaron en sus estimaciones de pobreza a partir de los resultados de la ronda de 2005 del Programa de Comparación Internacional (PCI) y de las extrapolaciones basadas en los resultados correspondientes a la ronda de 2011. Esto pone en evidencia la relevancia y sensibilidad que tienen esos cálculos.

Según lo señalado, es necesario dedicar recursos para disponer de PPA robustas, obtenidas a través de las rondas del PCI, al menos bajo un esquema de un criterio de referencia móvil (rolling benchmark) similar al empleado por Eurostat y la Organización para la Cooperación y el Desarrollo Económicos (OCDE). Mientras tanto, en la región se pueden utilizar las estimaciones que, pese a las limitaciones metodológicas que se señalan en las siguientes páginas, se reportan en este trabajo.

La literatura sobre los aspectos teóricos relacionados con las PPA es sumamente amplia. Existe también una vasta lista de publicaciones sobre los métodos de cálculo y los resultados puntuales obtenidos en las rondas del PCI, realizadas tanto en el ámbito académico (como es el caso de las Penn World Tables) como por organismos internacionales (Banco Mundial, ocDE, entre otros). Escasos son, en cambio, los trabajos de generación y análisis de series estadísticas regionales elaboradas a partir de metodologías complementarias a las utilizadas para calcular las PPA en los años en que se ejecutan las grandes operaciones estadísticas (rondas) de levantamiento de precios y cálculo de los ponderadores.

$\square$ Los autores, ex funcionarios de la Unidad de Estadísticas Económicas y Ambientales de la Comisión Económica para América Latina y el Caribe (CEPAL), agradecen las observaciones críticas y sugerencias formuladas por un árbitro anónimo a una versión previa de este trabajo.

1 Traducción propia.
El objetivo central de este trabajo es presentar algunos aspectos metodológicos y las series del PIB de América Latina y el Caribe para el período 2005-2013, expresadas en PPA, así como algunas limitaciones de ese ejercicio. Se llevan a cabo comparaciones con los resultados de la ronda de 2005 del PCI y con las series (a precios corrientes y constantes) expresadas en dólares, con tipos de cambio de mercado. Además, se formulan algunas hipótesis interpretativas acerca del comportamiento de las principales variables económicas calculadas en el marco de esta investigación.

A diferencia de un trabajo previo realizado por los autores, donde se calcularon las series (2000-2011) de las PPA sobre la base de los resultados de la ronda de 2005 del PCI, las series que se presentan en este nuevo estudio se basan en los resultados obtenidos en la ronda de 2011 del PCI publicados por la Oficina Mundial del Programa de Comparación Internacional (PCI), así como en las versiones más recientes de las cuentas nacionales difundidas por los países de América Latina y el Caribe respecto del período 2005-2013 (Epstein y Marconi, 2014).

En términos simples, la PPA entre dos países (A y B) es la relación entre el número de unidades de la moneda del país A requerido para comprar —en el país $\mathrm{A}$ - un producto de igual calidad y en igual cantidad que una unidad de la moneda del país B compraría en el país B. En este ejemplo, B es el país de referencia.

Mediante métodos econométricos, esas PPA —definidas inicialmente para productos elementalespueden calcularse para grupos de productos (denominados "encabezados básicos"), incluso a nivel del PIB y de sus componentes.

A partir de las PPA, se pueden analizar las diferencias de precios entre varios países y realizar comparaciones espaciales (dimensión geográfica), seleccionando a un país como referencia (generalmente a los Estados Unidos de América).

Las PPA son utilizadas para medir no solo el tamaño "real" de la economía de un país o de una región, sino tambien para obtener indicadores más robustos del nivel de desarrollo económico (como es el caso del PIB per cápita expresado en PPA), de productividad y competitividad, como asimismo medidas más adecuadas de pobreza.

En varios países de América Latina (Ecuador, El Salvador y Panamá) se ha adoptado la dolarización como 
régimen cambiario, perdiendo márgenes de acción en términos de política monetaria. Junto con el cálculo de tipos de cambio real, las series actualizadas de PPA permiten a las autoridades económicas de esos países disponer de útiles indicadores de competitividad.

Actualmente, las PPA son calculadas en el marco de un ejercicio coordinado por el Banco Mundial y ejecutado a nivel global mediante el PCI. Por su parte, la OCDE y Eurostat mantienen un programa regular y permanente mediante el cual realizan estos cálculos para sus países miembros.

Idealmente, este programa se podría llevar a cabo todos los años en todos los países del mundo, lo que permitiría disponer de series anuales de PPA. Lamentablemente, debido a restricciones financieras y logísticas, las PPA se disponen solo para los años de las rondas $^{2}$, aunque pueden ser estimadas para los años en que no se realizan esas grandes operaciones estadísticas a nivel mundial.

Académicos de la Universidad de Pennsylvania, liderados por Alan Heston, calculan series de PPA y de agregados macroeconómicos expresados en PPA para todos los países (Heston, varios años). A partir de 1996, utilizan los resultados de los PCI anteriores como referencia para estimar series largas, difundidas bajo el nombre de Penn World Tables (PWT). De manera similar a lo realizado en este trabajo, en las PWT se utiliza la información proveniente de las rondas del PCI como referente para obtener series de PPA.

Sin embargo, en muchos casos, la información que se incorpora en las PWT (así como la base de datos del Banco Mundial) no es la más actualizada para los países de América Latina y el Caribe, pues se trata de esfuerzos

2 Las dos últimas rondas del PCI tienen a 2005 y 2011 como años de referencia. a nivel global. Asimismo, en las extrapolaciones de PPA presentadas en las PWT se utilizan múltiples años de referencia obtenidos de las distintas rondas del PCI, mientras que el presente trabajo se focaliza en el último año disponible, pues se considera que posee la mejor calidad de información y metodologías de cálculo más avanzadas que las rondas precedentes.

En efecto, las estimaciones que se presentan en este estudio son realizadas a partir de la última información disponible del PCI, extrapolando (y retropolando) las paridades con los deflactores derivados de las cuentas nacionales de los países de América Latina y el Caribe y de los Estados Unidos de América, método que tiene la ventaja de su sencillez y bajo costo.

Se presentan las series del PIB a precios constantes y corrientes expresadas en PPA para los años 2005-2013, período acotado que, sin embargo, está influenciado estadísticamente por la crisis de los años 2008-2009, que pudo haber repercutido en los resultados presentados tanto en términos de precios como de volumen. Efectivamente, la crisis financiera desatada en esos años, el fin del ciclo de auge de los precios de los productos básicos (commodities) y la considerable disminución de la tasa de inversión registrados en los últimos años del período analizado, tuvieron un fuerte impacto en los precios relativos de los bienes y servicios que forman parte del PIB de los países de América Latina y el Caribe, en sus PPA y, evidentemente, en las bajas tasas de crecimiento del PIB total y per cápita en la mayoría de los países de la región.

Luego de esta Introducción, el artículo se desarrolla de la siguiente manera: en la sección II se ofrece una reseña de la metodología adoptada en este trabajo. A continuación, en la tercera sección se llevan a cabo comparaciones de los resultados obtenidos en el estudio. En tanto que, en la sección IV se ofrecen las consideraciones finales. Finalmente se agrega un anexo estadístico.

\section{II}

\section{Breve reseña metodológica}

El Sistema de Cuentas Nacionales (sCN, 2008, párrafos 15.232 y 15.233) hace referencia a los métodos de actualización de las PPA:

"El método comúnmente utilizado para extrapolar las PPA de su año de referencia a otro año es empleando el cuociente de los deflactores de cuentas nacionales de cada país comparado con un país numerario para proyectar las PPA de cada país a partir del año de referencia".

"En teoría, la mejor forma de extrapolar las PPA a partir de un año de referencia sería utilizar series de tiempo de precios a nivel de productos individuales. En la práctica, no es posible, [...] por lo tanto, en general se adopta un enfoque basado 
en la extrapolación a un nivel macro (para el PIB o algunos componentes del PIB)" (CE/FMI/OCDE/ Naciones Unidas/Banco Mundial, 2009).

El SCN 2008 recomienda utilizar un año de referencia en el que las PPA son robustas (es decir, un año en que se haya realizado una ronda del PCI); a partir de dicho año, se procede a extrapolar (a retropolar o ambos) esos niveles empleando los deflactores calculados en cuentas nacionales.

Sin embargo, se presenta un problema metodológico en relación con el nivel de desagregación. Efectuar la extrapolación a nivel de productos individuales, como se propone en el párrafo 15.233 del SCN 2008, supone llevar a cabo un programa de comparación a nivel mundial que posibilite disponer (con las limitaciones típicas de un proyecto de esa naturaleza) de la información requerida.

Obviamente, si la información se encuentra disponible, lo ideal es realizar extrapolaciones al mayor nivel de detalle, por ejemplo, por medio de grandes componentes del gasto del PIB o incluso a nivel de ciertos encabezados básicos (basic headings). La elección del nivel al que se realiza la extrapolación depende principalmente de la cantidad y calidad de la información disponible, tanto en lo que se refiere a las cuentas nacionales como a los precios de los bienes y servicios de una canasta homogénea de productos ${ }^{3}$.

${ }^{3}$ Una exposición resumida sobre los métodos de agregación utilizados en el marco del PCI se presentan en Epstein y Marconi (2014).
Como se señaló, para construir las series correspondientes a 2005-2013 se utilizaron los resultados de la ronda de 2011 publicados por el Banco Mundial, así como la información publicada en CEPALSTAT (disponible a enero de 2015) para retropolar (2005-2010) y extrapolar (2012-2013) los valores de las PPA. La retro/ extrapolación de las PPA de 2011 se realizó de acuerdo con las recomendaciones del SCN 2008, en función de la disponibilidad de los deflactores implícitos y de las PPA a nivel del PIB, utilizando la siguiente fómula:

$$
P P A_{t+k}^{A}=P P A_{t}^{A} \times \frac{D I_{t+k}^{A}}{D I_{t+k}^{R}}
$$

en la que $P P A_{t}^{A}$ es la PPA para el país A, en el período $t$; $D I_{t+k}^{A}$ es el deflactor implícito del PIB en el país A, en el período $t+k$ (base $=100$ para el período $t$ ); y $D I_{t+k}^{R}$ es el deflactor implícito del PIB del país de referencia $\mathrm{R}$, en el período $t+k$ (base $=100$ para el período $t$ ). En el anexo se reportan los principales resultados.

Cabe hacer notar, sin embargo, que en el cálculo de los deflactores de cuentas nacionales se utilizan en los denominadores estructuras que varían en el tiempo, lo que obviamente puede dificultar la comparación. Idealmente, estos deflactores deberían ser obtenidos a partir de una canasta fija en el año de referencia.

Una vez calculadas las series de PPA para el período, se obtuvieron las series del PIB dividiendo los valores a precios corrientes en moneda nacional (MN) por las

\footnotetext{
\begin{tabular}{rr}
\hline Recuadro 1 \\
\hline
\end{tabular}

LA TEORÍA ECONÓMICA DETRÁS DE LAS PPA

El concepto de paridad del poder adquisitivo surge en el siglo xvi bajo la forma de una Ley del Precio Único, que establece que los precios de dos bienes exactamente iguales en dos países distintos deben ser iguales en el largo plazo, debido a la existencia de un arbitraje internacional. De esta forma, si el precio de un bien sube más en un país que en el otro, el tipo de cambio entre las monedas deberá variar proporcionalmente para mantener la paridad internacional.

Extendiendo esa idea a un grupo de bienes, el índice de las PPA para toda la economía debería ser igual a la tasa de cambio en el largo plazo.

Sin embargo, existen razones por las cuales la Ley del Precio Único y, por tanto, la paridad entre las PPA y los tipos de cambio, no se cumple debido a la existencia de costos de transporte, impuestos o tarifas comerciales, o a causa de la no transabilidad de ciertos servicios entre países.

Por otra parte, existe otro tipo de hipótesis teóricas que pueden explicar las diferencias en los niveles de precios entre los países. En la denominada hipótesis Balassa-Samuelson se establece que aquellos países con una mayor productividad (y, por tanto, mayor ingreso) presentarán niveles de precios más altos. Una consecuencia de esta hipótesis es el llamado "efecto Penn", a través del cual aquellos países con un PIB mayor presentarán un índice de nivel de precios (calculado como la razón entre las PPA y el tipo de cambio) sistemáticamente mayor, y viceversa. Esto indicaría también que, a medida que un país se desarrolla y su riqueza relativa se incrementa, también aumentan sus precios relativos.
}

Fuente: Elaboración propia. 
correspondientes PPA estimadas de cada año entre 2005 y 2013. De esta forma, se obtienen las series expresadas en PPA a precios corrientes en dólares de los Estados Unidos de América, para 30 países de América Latina y el Caribe 4 .

Por su parte, el índice de nivel de precios (Price Level Index — PLI - por su nombre y sigla en inglés), se define de la siguiente forma:

$$
P L I_{t}^{k}=\frac{P P A_{t}^{k}}{E_{t}^{k}}
$$

donde $P L I_{t}^{k}$ es el índice de nivel de precios para el país $k$ en el año $t$; y $E_{t}^{k}$ es el tipo de cambio de mercado del país $k$ en el año $t$ con respecto al país de referencia, en este caso, los Estados Unidos de América.

Este índice es un porcentaje que indica el nivel de precios en el país $k$ con respecto al nivel de precios en los Estados Unidos de América, considerando al dólar como moneda de referencia. Por lo tanto, un valor del PLi igual a 0,8 (u 80\%) significa que en el país $k$, con 80 centavos, se puede comprar lo mismo que se compraría en los Estados Unidos de América con un dólar. Alternativamente, se puede señalar que el nivel de precios es $20 \%$ menor en el país $k$ que en los Estados Unidos de América.

Finalmente, el cálculo de las series del PIB, a precios constantes, expresado en PPA, se realizó considerando 2010 como año de referencia. Para obtener estas series, se partió de los valores del PIB en el año 2010 a PPA corrientes, extra/retropolándolos para el período 2005-2013 con las correspondientes tasas de variación de las series del PIB de cada país, a precios constantes ${ }^{5}$.

En este ejercicio, las series se expresan a precios (de los Estados Unidos de América) de 2010, que por lo demás es el año base de las series regionales difundidas por la CEPAL.

\footnotetext{
${ }^{4}$ Los países no cubiertos por este trabajo son la Argentina, Cuba y Guyana. Se decidió no incluir a Cuba en el estudio, pues la Oficina Global, en la difusión de los resultados de la ronda de 2011 del PCI, incorpora la siguiente nota: "El PIB oficial de Cuba para el año de referencia 2011 es de 68.999,15 millones, en moneda nacional. Sin embargo, este valor y su desagregación no se incluyen en las tablas debido a asuntos de comparabilidad metodológica. Por lo tanto, solo se presentan los valores de PPA y el índice de nivel de precios de Cuba" (World Bank [online] http://icp.worldbank.org).

Por otra parte, la Argentina y Guyana se autoexcluyeron de la ronda de 2011.

5 Esto equivale a utilizar la serie a precios constantes en moneda nacional para el período 2005-2013 y dividir los valores por la PPA del año escogido como referencia.
}

Dalgaard y Sorensen (2002) advierten que el método agregado puede introducir sesgos en las estimaciones si las estructuras de las economías de los países se modifican en el tiempo, de modo que la única manera de mitigar ese efecto es realizar la extrapolación al mayor nivel de desagregación posible.

Con el propósito de evaluar los resultados y comparar los dos enfoques metodológicos (PIB en su mayor nivel de agregación comparado con su desagregación) se realizó un ejercicio de cálculo de las PPA por componentes. El nivel de los componentes corresponde a las PPA publicadas por el PCI en la ronda de 2011: gasto de consumo individual de gobierno, gasto de consumo colectivo de gobierno, gasto de consumo de los hogares, formación bruta de capital, exportaciones e importaciones de bienes y servicios. Para cada uno de ellos, se utilizaron los deflactores implícitos del PIB correspondientes con el fin de extra/retropolar las PPA del año de referencia 2011. A partir de estas series de PPA por componente, se obtuvieron las PPA a nivel del PIB a través del método EKs de agregación, para cada uno de los años en el período de referencia.

La información fue obtenida a partir de la base de datos de la CEPAL (CEPALSTAT) ${ }^{6}$ y, en el caso del país base (Estados Unidos de América), del sitio web de la Oficina de Análisis Económicos (www.bea.gov) ${ }^{7}$. Para las importaciones y exportaciones de bienes y servicios, se usaron como PPA las tasas de cambio de cada año, recomendación metodológica propuesta en el contexto del PCI.

Conviene reiterar que para eliminar los sesgos mencionados por Dalgaard y Sorensen (2002), es necesario realizar la extrapolación a nivel de producto (y no de componente). Por lo tanto, en los resultados obtenidos en este estudio se incorporan aun esos sesgos, aunque menores si se comparan los resultados con la extrapolación a nivel agregado del PIB.

\footnotetext{
${ }^{6}$ En el caso del consumo del gobierno general, es necesario trabajar con su desglose en consumo colectivo e individual, y calcular por separado los respectivos deflactores, pues ese es el nivel de desagregación publicado por el PCI en 2011. Sin embargo, debido a la falta de información respecto de la mayoría de los países, se decidió utilizar el deflactor del total del consumo del gobierno general en el cálculo de estos dos componentes.

${ }^{7}$ Cabe señalar que no se encuentran disponibles para los Estados Unidos de América datos del consumo del gobierno general desglosado en sus componentes: colectivo e individual y, por lo tanto, fue necesario realizar estimaciones basadas tanto en información publicada en las rondas del PCI, como en datos auxiliares publicados por los países.
} 


\section{III}

\section{Principales resultados}

Varias son las comparaciones que pueden realizarse con los resultados obtenidos en este estudio. A continuación se presentan las principales diferencias en la clasificación de los países de la región cuando el PIB per cápita se expresa en tipos de cambio de mercado y en PPA para los años de referencia (2005 y 2011). Se comparan también las series del PIB total y per cápita expresadas en PPA y en tipos de cambio de mercado, tanto a precios corrientes como constantes, como también los niveles de precios, poniendo de esa manera en evidencia a los países más "caros" o más "baratos" de la región. Además, se reportan algunos elementos que se desprenden de la comparación entre los resultados obtenidos con el método de los componentes y, finalmente, se analizan algunos elementos que deben tomarse en consideración cuando se realiza este tipo de comparaciones.

\section{Comparación estática: clasificación de países en 2005 y 2011}

En el gráfico 1 se presentan las diferencias en la clasificación cuando se emplean los tipos de cambio del mercado (XR) y cuando se deflacta con PPA para 2005 y 2011. Las PPA utilizadas en el cálculo son aquellas publicadas en el marco del PCI respecto de las rondas correspondientes; por esta razón, solo se incluyen resultados para los diez países participantes en ambas rondas: Bolivia (Estado Plurinacional de), Brasil, Chile, Colombia, Ecuador, México, Paraguay, Perú, Uruguay y Venezuela (República Bolivariana de).

Si se emplea el tipo de cambio (XR), México es el país con mayor PIB per cápita en 2005, seguido por Chile. Por otra parte, si se utilizan las PPA ese orden se invierte. Esto se debe a que el nivel de precios es menor en Chile que en México.

De forma similar, existen importantes diferencias en la clasificación con respecto al año 20: el Brasil es el país que más cambia relativamente, pasando del tercer puesto (cuando se utiliza el XR) al quinto lugar cuando se deflacta con la PPA. Es importante notar que en el año 2011 se registró una marcada apreciación del real, alcanzando una tasa de cambio de 1,67 por dólar, el valor más bajo en el período 2000-2013. Esto tuvo un efecto directo en el PIB per cápita nominal en dólares del Brasil en ese año, que se neutraliza cuando se utiliza la PPA como deflactor.

Cuatro hipótesis interpretativas podrían formularse sobre las diferencias en el nivel de precios de los países de la región, las que deberán ser estudiadas y verificadas oportunamente. La primera consiste en los diferenciales existentes en el grado de apertura comercial y arancelaria de los países de la región; la segunda dice relación con los niveles de concentración de los mercados y diferenciación de los productos que forman parte de la "canasta del PIB". Un tercer elemento no indiferente en la determinación y evolución de los precios es el efecto de las políticas monetarias y fiscales, así como la manera en que los agentes económicos forman (anticipan) sus expectativas. Finalmente, pero no menos importante, la existencia de brechas de productividad, es decir, la distinta dinámica en los procesos de generación y de apropiación del excedente (surplus) $)^{8}$ puede influir notablemente en la configuración de los precios (absolutos y relativos) y en su dinámica intertemporal.

Hay que subrayar que cuando se pasa de 2005 a 2011 (es decir, lectura vertical del gráfico), la clasificación en que se que utilizan las PPA como tasa de conversión es más estable que aquella obtenida al emplear el XR. Esto se puede apreciar, por ejemplo, en los cambios que experimentan México y el Brasil, los dos países más grandes de la región. Al usar el XR, México pasa del primer al quinto puesto, mientras que el Brasil "salta" del quinto al tercero, cambios que responden a la variación de los precios relativos (cf. supra). Mientras que México pasa del segundo (año 2005) al cuarto puesto (ronda de 2011), el Brasil se mantiene en el quinto puesto en ambos años.

\footnotetext{
${ }^{8}$ Este último aspecto está siendo estudiado por parte de los autores en el marco de una investigación basada en el método del excedente (surplus), aplicado a los países de América Latina.
} 
GRÁFICO 1

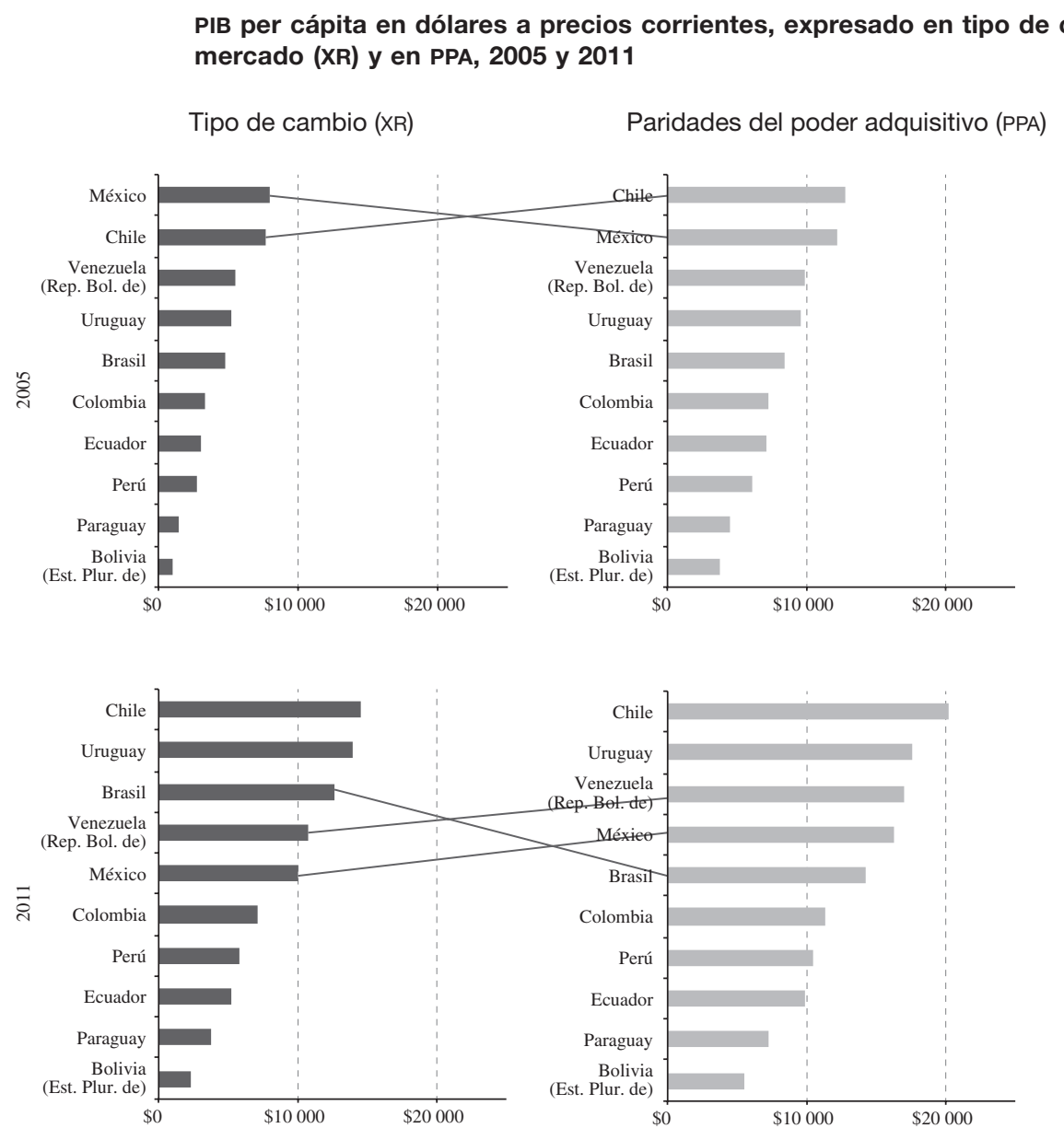

Fuente: Elaboración propia sobre la base de Comisión Económica para América Latina y el Caribe (CEPAL), Bases de Datos y Publicaciones Estadísticas (CEPALSTAT) [en línea] http://estadisticas.cepal.org/; y Base de Datos del Banco Mundial [en línea] http://data.worldbank.org/. Nota: PIB: Producto interno bruto.

\section{Comparación dinámica: evolución del PIB expresada en PPA y en tipos de cambio de mercado}

Una de las principales ventajas de calcular series del PIB a PPA es la de permitir la evaluación de las contribuciones reales de los países a las economías regionales, es decir, el tamaño de las economías. En el gráfico 2 se observan estas participaciones a lo largo del período estudiado respecto de los dos países más grandes de la región (Brasil y México) en términos del PIB total de América
Latina (expresadas de acuerdo con las series a precios corrientes $)^{9}$.

Si se calculan las participaciones en términos de tipo de cambio nominal en el año 2005, las economías de México y el Brasil tienen el mismo tamaño (ponderación de $32 \%$ cada una de ellas en el total regional), mientras

\footnotetext{
9 En el contexto de este trabajo, la región de América Latina y el Caribe incluye solo a los países para los cuales se pudieron realizar los cálculos relevantes. Por lo tanto, no incorpora a la Argentina y Cuba por las razones señaladas.
} 
Proporción del PIB del Brasil y México con respecto al PIB regional: PPA comparado con el XR (a precios corrientes)

(En porcentajes)

Brasil

Proporción del PIB regional

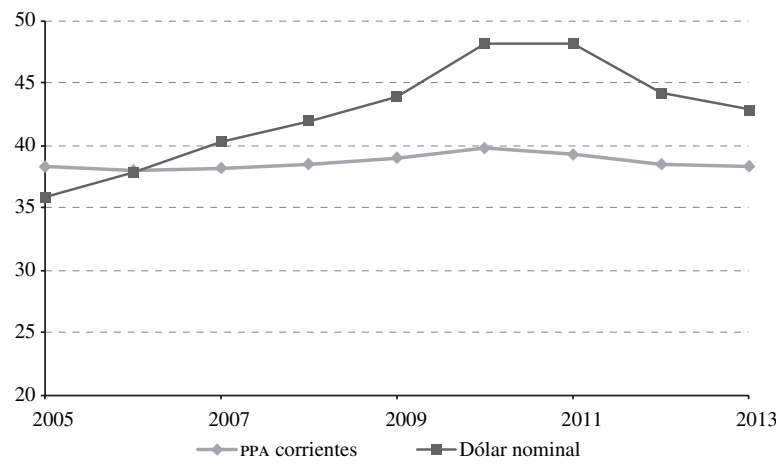

México

Proporción del PIB regional

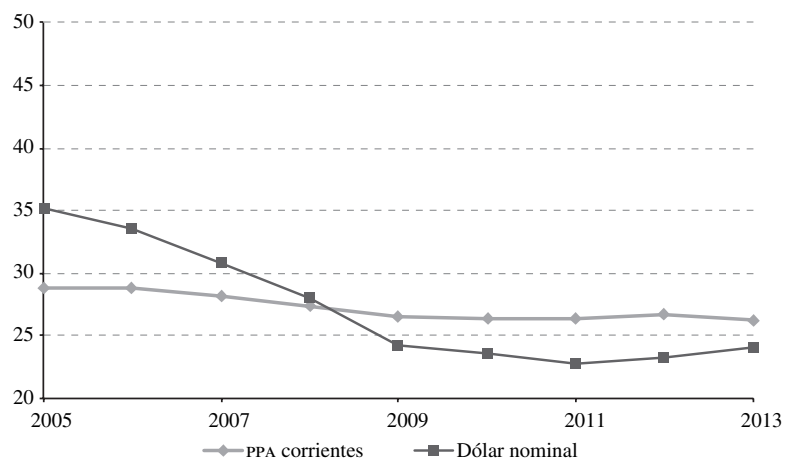

Fuente: Elaboración propia sobre la base de Comisión Económica para América Latina y el Caribe (CEPAL), Bases de Datos y Publicaciones Estadísticas (CEPALSTAT) [en línea] http://estadisticas.cepal.org/; y Base de Datos del Banco Mundial [en línea] http://data.worldbank.org/. Nota: XR: Tipo de cambio de mercado; PIB: Producto interno bruto; PPA: Paridades del poder adquisitivo.

que en términos de PPA, los resultados indican que el Brasil (38\% del PIB total regional) es significativamente más grande que México (29\%).

Como se puede apreciar, la participación del Brasil en la economía regional crece en términos nominales, pasando de $32 \%$ en 2005 a $38 \%$ del PIB total regional en 2013, alcanzando al $43 \%$ en 2010 y 2011. Sin embargo, al tener en cuenta el nivel de precios relativos del país, esta proporción se mantiene entre el 38\% y el $40 \%$ para todo el período de referencia. Esto se debe tanto al aumento del nivel de precios del Brasil con respecto al resto de los países de la región, como a las grandes variaciones de tipo de cambio registradas durante el período analizado.

En el caso de México, país cuya participación en términos nominales es similar a la del Brasil en 2005 (32\%), su ponderación se reduce pronunciadamente, estabilizándose en alrededor del $21 \%$ a partir de 2009. Esto obedece al hecho de que México, hasta 2007, fue uno de los países "más caros" de la región, aunque su nivel de precios no creció tanto como el de los demás países grandes de América Latina. Así, entre los cinco países con las economías más grandes de la región (en términos del PIB total) cubiertos por este estudio ${ }^{10}$, México fue el más caro hasta 2007, mientras que a partir

${ }^{10}$ Brasil, México, Colombia, Venezuela (República Bolivariana de) y Chile. de 2011 resultó ser el "más barato". Por otra parte, al considerar las series expresadas en PPA, la participación de México muestra una leve tendencia negativa, cayendo del $29 \%$ en 2005 al $26 \%$ en 2013.

Cabe destacar que las participaciones en términos de PPA se muestran más estables y con menor variabilidad que las nominales, característica que se registra no solo para estos dos casos, sino para todos los países de la región ${ }^{11}$.

En el gráfico 3 se muestra la evolución del PIB per cápita para América Latina y el Caribe, así como la diferencia entre ambas subregiones respecto de las series expresadas en PPA y en tipos de cambio de mercado (XR).

$\mathrm{Al}$ analizar la serie de XR en dólares corrientes, se puede apreciar que a partir de 2009, las dos subregiones se acercan en términos de PIB per cápita, manteniendo valores muy similares desde 2011. Tratándose de valores corrientes (XR), gran parte de ese cambio puede explicarse por diferencias en los precios relativos.

En el gráfico 4 se advierte la evolución de los deflactores del PIB para ambas regiones en el período y puede apreciarse claramente la diferencia en sus tendencias, especialmente a partir de 2009, cuando se evidencia una aceleración de los precios relativos en América Latina con respecto al Caribe. Esto pone en

${ }^{11}$ En el anexo estadístico se presentan las series del PIB a PPA de los países de América Latina y el Caribe actualizadas con información hasta enero de 2015, a partir de las cuales es posible realizar un análisis para cada uno de los países de la región. 
evidencia el efecto Balassa-Samuelson, pues esta aceleración ocurre en la región menos afectada por la crisis de 2009 y, por lo tanto, aquella que presentó un mayor crecimiento relativo de su PIB.

GRÁFICO 3

PIB per cápita de América Latina y el Caribe en dólares a precios corrientes, en PPA y en XR, 2005-2013

PIB per cápita en XR, dólares (a precios corrientes)

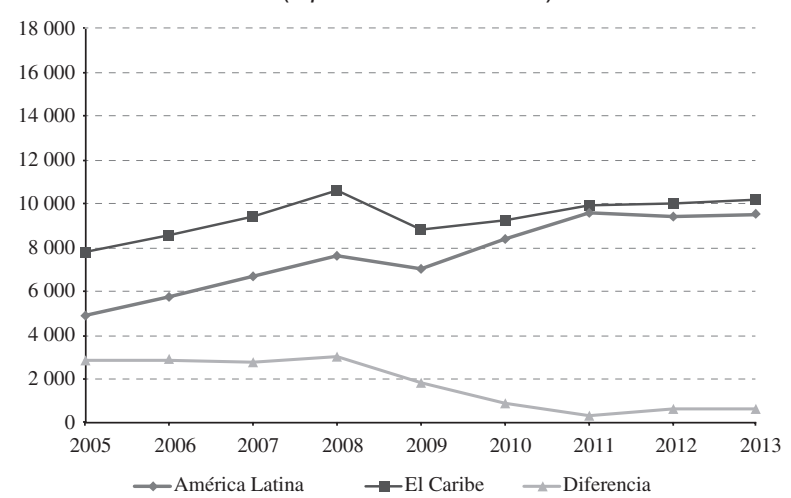

PIB per cápita en XR, corrientes (PPA corrientes)

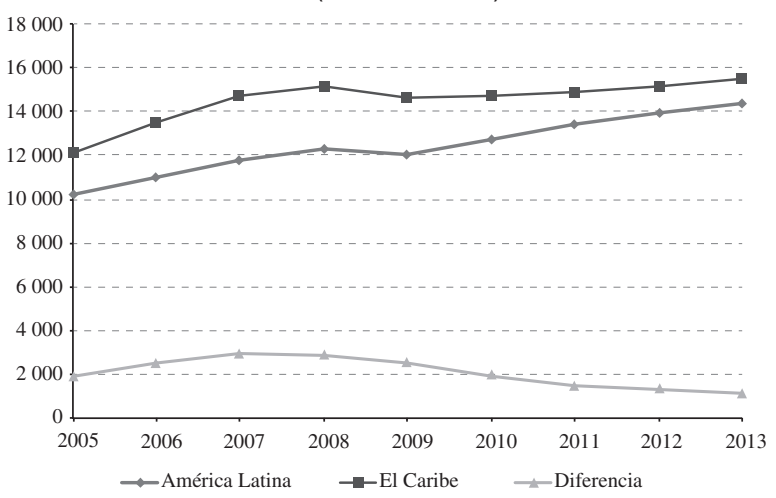

Fuente: Elaboración propia sobre la base de Comisión Económica para América Latina y el Caribe (CEPAL), Bases de Datos y Publicaciones Estadísticas (CEPALSTAT) [en línea] http://estadisticas.cepal.org/; y Base de Datos del Banco Mundial [en línea] http://data.worldbank.org/. Nota: PPA: Paridades del poder adquisitivo; PIB: Producto interno bruto; XR: Tipo de cambio de mercado.

GRÁFICO 4

Deflactores del PIB para América Latina y el Caribe, 2005-2013 $(2005=100)$

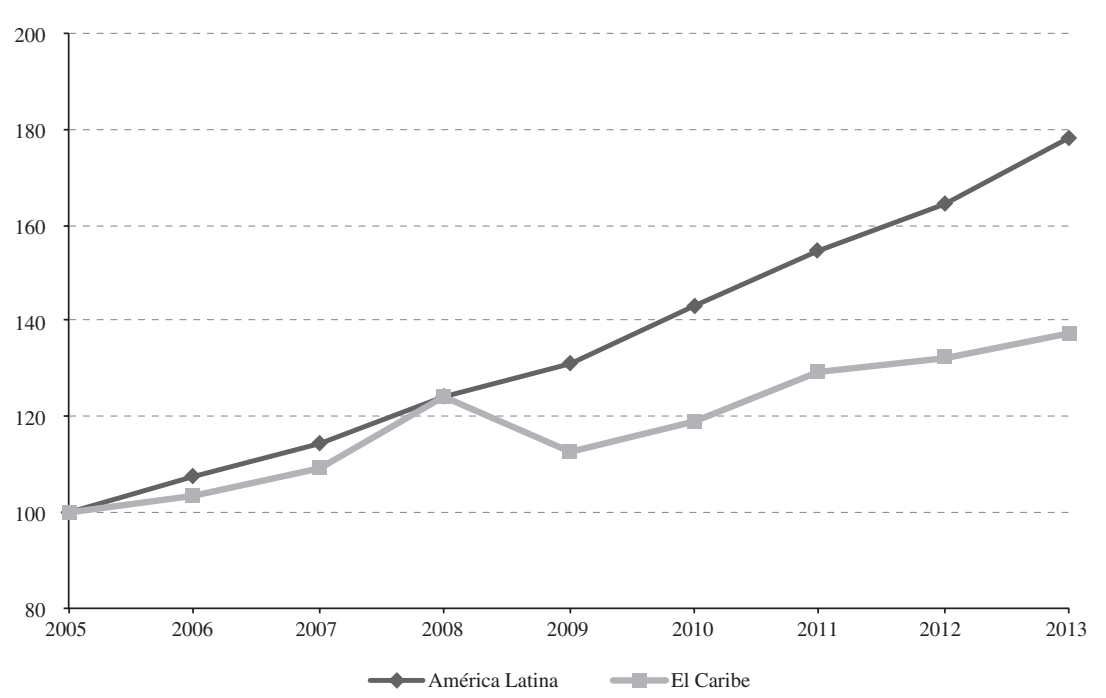

Fuente: Elaboración propia sobre la base de Comisión Económica para América Latina y el Caribe (CEPAL), Bases de Datos y Publicaciones Estadísticas (CEPALSTAT) [en línea] http://estadisticas.cepal.org/; y Base de Datos del Banco Mundial [en línea] http://data.worldbank.org/. Nota: PIB: Producto interno bruto. 
Al comparar el índice de nivel de precios (PLI) con el ingreso relativo de cada país (véase el gráfico 5) medido sobre la base del índice del PIB nominal per cápita con respecto al total regional, se puede observar cierta correlación positiva entre ambas variables. Si bien la relación no es muy marcada, pone en evidencia el efecto Penn y la hipótesis Balassa-Samuelson, pues aquellos países con mayor nivel de ingreso muestran en general un más elevado nivel de precios. Por esta razón, la medición del PIB en PPA tiende a acortar las brechas en términos de PIB per cápita frente a las comparaciones en valores nominales.

En cuanto a las series a precios constantes, estas permiten un estudio simultáneo intertemporal e interespacial, a diferencia de las series a PPA corrientes en las que los resultados deben tratarse más bien como un panel, posibilitando realizar comparaciones de tipo cross section para los años disponibles. Sin embargo, cabe recordar que la metodología utilizada presenta la desventaja de que tanto los niveles de las series como las tasas de crecimiento regionales o subregionales pueden variar significativamente en función del año escogido como base y de los países incorporados en el análisis ${ }^{12}$.

12 La autoexclusión de un país grande como la Argentina de la última ronda del PCI tiene obvias repercusiones en los resultados del estudio, tanto en términos de ponderación como en el nivel y
No se analizan las tasas de crecimiento de los países a lo largo del período, pues son las calculadas a partir de las series a precios constantes difundidas por las instituciones nacionales encargadas de elaborar las cuentas nacionales. En cambio, vale la pena analizar los resultados a nivel regional y subregional. En el gráfico 6 se presenta el PIB per cápita expresado en PPA, a precios constantes de 2010.

Las series del PIB per cápita expresadas a PPA constante de América Latina y del Caribe son muy similares al caso de las PPA corrientes (véase el gráfico 3). Esto se debe a que, dado el método de extrapolación de las PPA utilizado con los deflactores implícitos, en las PPA corrientes se incorpora el efecto de precios relativos con respecto a los Estados Unidos de América.

Por otra parte, al analizar las series del PIB a PPA constantes para las cuatro subregiones, se puede apreciar que la crisis de 2009 afectó en mayor medida a la oECS (Antigua y Barbuda, Dominica, Granada, Saint Kitts y Nevis, San Vicente y las Granadinas y Santa Lucía) que al resto de países. De hecho, el valor per cápita al comienzo del período (2005) es similar al de 2013 (12.413 dólares y 12.499 dólares, respectivamente). El

evolución de las PPA regionales. Al respecto, Epstein y Marconi (2014) presentan un análisis del efecto de la incorporación de este país en los cálculos.

GRÁFICO 5

Relación entre el nivel del PIB per cápita y el nivel de precios de los países de América Latina y el Caribe, 2011

(Eje horizontal, región =100; eje vertical, Estados Unidos de América =100)

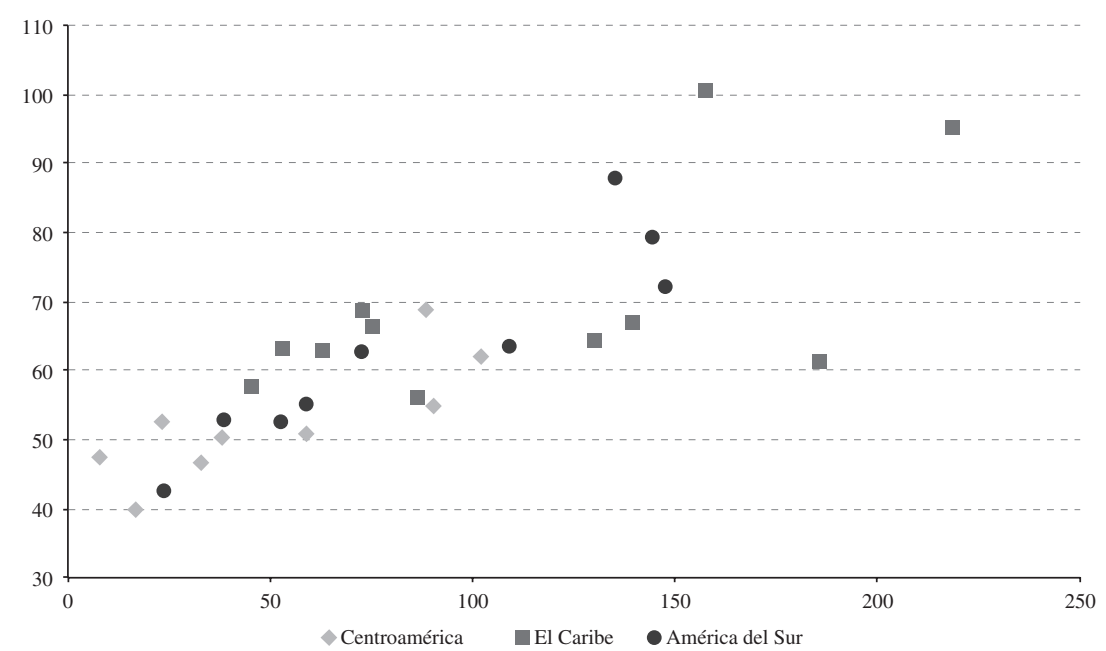

Fuente: Elaboración propia sobre la base de Comisión Económica para América Latina y el Caribe (CEPAL), Bases de Datos y Publicaciones Estadísticas (CEPALSTAT) [en línea] http://estadisticas.cepal.org/, y Base de Datos del Banco Mundial [en línea] http://data.worldbank.org/. Nota: PIB: Producto interno bruto. 
GRÁFICO 6

PIB per cápita en dólares (PPA), a precios constantes para América Latina y el Caribe, 2005-2013

(Año base $=2010$ )
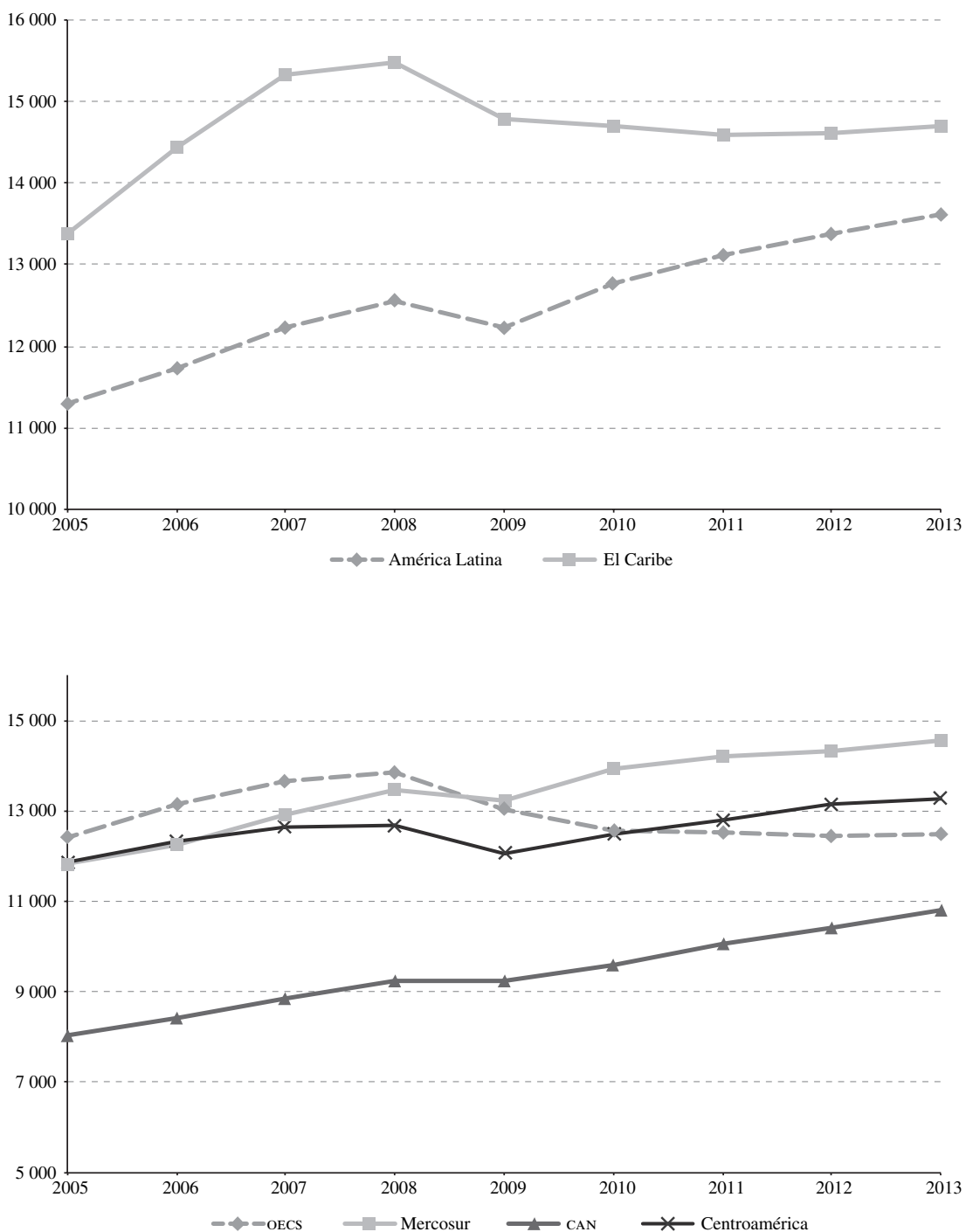

Fuente: Elaboración propia sobre la base de Comisión Económica para América Latina y el Caribe (CEPAL), Bases de Datos y Publicaciones Estadísticas (CEPALSTAT) [en línea] http://estadisticas.cepal.org/, y Base de Datos del Banco Mundial [en línea] http://data.worldbank.org/. Nota: oEcs: Antigua y Barbuda, Dominica, Granada, Saint Kitts y Nevis, San Vicente y las Granadinas y Santa Lucía. Mercosur: Brasil. Paraguay, Uruguay y Venezuela (Rep. Bol. de). CAN: Bolivia (Est. Plur. de), Colombia, Ecuador y Perú. Centroamérica: Costa Rica, El Salvador, Guatemala, Haití, Honduras, México, Nicaragua, Panamá y República Dominicana. PIB: Producto interno bruto.

PIB de esa subregión superaba al de las otras tres subregiones en 2005, pero se ubicó en el tercer puesto en 2013, solo superando a la Comunidad Andina de Naciones (CAN). Contrariamente, el Mercado Común del Sur (MERCOSUR) logró crecer notablemente durante el período (23\% acumulado), pasando de 11.835 dólares en 2005 a 14.539 dólares en 2013, lo que lo ubicó como la subregión con el mayor PIB per cápita (expresado en PPA constantes de 2010).

Por su parte, Centroamérica también sufrió el efecto de la crisis, presentando un nivel muy similar al del MERCOSUR en 2005 (11.868 dólares), y registrando un 
crecimiento acumulado de $11 \%$ entre 2005 y 2013. Finalmente, la CAN se mantuvo durante todo el período como la subregión con el menor PIB per cápita en términos de PPA constantes, a pesar de haber sido la subregión con el más rápido crecimiento, pasando de 8.040 dólares en 2005 a 10.814 dólares en 2013 (con un incremento acumulado de $35 \%$ ).

Finalmente, las diferencias entre las series expresadas en tipos de cambio de mercado y aquellas en PPA pueden explicarse por la evolución del índice de nivel de precios según el Programa de Comparación Internacional (PLI). En el cuadro 1 se muestran los valores que arroja el PLI con respecto al PIB total para los países de la región, expresados en términos del nivel de precios de los Estados Unidos de América.

El cuadro 1 permite observar tanto la evolución del PLI, relativo al nivel de precios de los Estados Unidos de América (comparación horizontal en el cuadro 1), como las relaciones entre países para un año determinado (comparación vertical).

Cuando se considera la evolución de los índices del PLI, se puede apreciar que América del Sur (60\% acumulado) es la subregión cuyos precios crecieron más respecto de aquellos de los Estados Unidos de América, con países cuyos niveles en el PLI varían entre el 37\% y el $90 \%$ acumulado durante el período. El Uruguay, por ejemplo, pasó de un nivel de precios menor a la mitad del de los Estados Unidos de América en 2005, a más del $80 \%$ en 2013. El análisis por componente del PIB pone en evidencia el fuerte incremento del deflactor del gasto del gobierno (incremento salarial) en ese país, en comparación con los demás.

A su vez, Centroamérica (6\%) mostró un aumento importante aunque no alcanza los niveles de América del Sur, con países cuyos niveles de precio varían entre el $15 \%$ y el $50 \%$ acumulado durante el período con respecto a los niveles de los Estados Unidos de América, con excepción de México, El Salvador y la República
Dominicana, que mostraron variaciones de un $4 \%$, un $8 \%$ y $-2 \%$, respectivamente.

Por último, el Caribe (2\%) presenta leves incrementos, e incluso registra algunas disminuciones, con excepción de Suriname, cuyo movimiento de precios es más similar al de la región sudamericana.

En lo que se refiere a la comparación "vertical" entre países, los del Caribe muestran niveles de precios relativos muy similares, con excepción de las Bahamas y Barbados, que son incluso más caros que los Estados Unidos de América en varios años del período analizado (PLI mayor que 100). Por su parte, Centroamérica muestra niveles relativamente similares, con excepeción de Costa Rica y México, que son los países más caros de la subregión hacia finales del período 2005-2013. En este sentido, Costa Rica es el país que más cambia su posición relativa en lo referido a los niveles del PLI, pasando del cuarto lugar en 2005 a ser el más caro de la subregión en 2013, superando ampliamente a México (más de diez puntos porcentuales). La explicación, al igual que el caso del Uruguay, puede encontrarse en las políticas de incremento de remuneraciones en el sector público (véase el gráfico 7).

América del Sur presenta mayor heterogeneidad en cuanto al nivel de precios relativos. Si, por ejemplo, se considera a países como el Brasil, Colombia y el Uruguay, sus niveles de precios en 2005 eran muy similares. Sin embargo, estos niveles son muy diferentes hacia el final del período (diferencias de alrededor de 20 puntos porcentuales en 2013). Por su parte, Chile era en 2005 el país más caro de la subregión, superando a Venezuela (República Bolivariana de) en casi diez puntos porcentuales. En 2013, los niveles de Chile y Venezuela (República Bolivariana de) se equiparan en alrededor del 70\% del nivel de precios de los Estados Unidos de América, ubicándose como el tercer y cuarto país más caro de la subregión, respectivamente, después del Uruguay y el Brasil. 
CUADRO 1

Índices de nivel de precios, 2005-2013

(Estados Unidos de América $=100$ )

\begin{tabular}{|c|c|c|c|c|c|c|c|c|c|c|}
\hline Región o país & 2005 & 2006 & 2007 & 2008 & 2009 & 2010 & 2011 & 2012 & 2013 & $\begin{array}{c}\text { Variación } \\
\text { Porcentual } \\
2005-2013\end{array}$ \\
\hline \multicolumn{11}{|l|}{ Centroamérica } \\
\hline Costa Rica & 48,8 & 49,1 & 51,8 & 56,1 & 55,4 & 64,4 & 68,6 & 70,5 & 73,4 & 50 \\
\hline El Salvador & 45,8 & 46,4 & 47,2 & 48,7 & 48,1 & 48,6 & 50,3 & 49,9 & 49,3 & 8 \\
\hline Guatemala & 37,2 & 38,1 & 39,4 & 42,9 & 40,8 & 43,0 & 46,6 & 47,0 & 47,7 & 28 \\
\hline Haití & 32,2 & 35,9 & 41,5 & 43,3 & 42,3 & 45,6 & 47,2 & 47,1 & 47,8 & 48 \\
\hline Honduras & 40,2 & 40,9 & 42,5 & 44,9 & 48,0 & 49,7 & 52,5 & 51,7 & 49,6 & 23 \\
\hline México & 58,7 & 60,5 & 61,7 & 63,1 & 53,3 & 58,8 & 61,8 & 59,1 & 61,0 & 4 \\
\hline Nicaragua & 34,2 & 34,2 & 34,7 & 37,7 & 38,0 & 38,7 & 39,8 & 40,0 & 39,9 & 17 \\
\hline Panamá & 49,4 & 48,9 & 49,1 & 51,9 & 51,7 & 53,6 & 54,7 & 55,6 & 56,8 & 15 \\
\hline \multirow[t]{2}{*}{ República Dominicana } & 49,0 & 45,7 & 47,1 & 49,0 & 48,4 & 49,3 & 50,9 & 50,5 & 48,2 & -2 \\
\hline & 56,0 & 57,5 & 58,6 & 60,2 & 52,1 & 57,0 & 59,9 & 57,8 & 59,3 & 6 \\
\hline \multicolumn{11}{|l|}{ El Caribe } \\
\hline Antigua y Barbuda & 63,4 & 61,7 & 62,3 & 63,9 & 64,5 & 64,6 & 64,1 & 64,6 & 63,5 & 0 \\
\hline Bahamas & 103,5 & 101,2 & 101,5 & 101,0 & 99,2 & 97,6 & 94,9 & 95,6 & 95,9 & -7 \\
\hline Barbados & 105,3 & 107,2 & 107,4 & 105,7 & 110,6 & 105,2 & 100,8 & 95,6 & 96,5 & -8 \\
\hline Belice & 56,1 & 56,9 & 58,1 & 58,5 & 56,3 & 56,3 & 57,5 & 57,6 & 57,7 & 3 \\
\hline Dominica & 67,3 & 66,9 & 66,2 & 66,1 & 70,6 & 68,2 & 68,9 & 69,5 & 69,4 & 3 \\
\hline Granada & 63,8 & 64,8 & 64,5 & 68,3 & 67,8 & 67,3 & 66,0 & 67,4 & 67,8 & 6 \\
\hline Jamaica & 63,0 & 63,1 & 56,3 & 59,6 & 54,7 & 59,9 & 63,0 & 63,9 & 60,2 & -4 \\
\hline Saint Kitts y Nevis & 58,9 & 63,2 & 64,4 & 65,2 & 66,1 & 65,9 & 66,8 & 66,8 & 66,1 & 12 \\
\hline San Vicente y las Granadinas & 61,0 & 60,9 & 64,3 & 63,1 & 62,1 & 64,1 & 62,6 & 62,3 & 61,8 & 1 \\
\hline Santa Lucía & 62,5 & 63,9 & 67,1 & 65,1 & 64,5 & 68,1 & 68,3 & 68,9 & 69,9 & 12 \\
\hline Suriname & 44,3 & 45,1 & 46,8 & 53,0 & 56,0 & 59,3 & 55,9 & 60,4 & 61,1 & 38 \\
\hline \multirow[t]{2}{*}{ Trinidad y Tabago } & 54,0 & 52,7 & 57,9 & 70,7 & 50,5 & 53,4 & 61,4 & 58,8 & 60,0 & 11 \\
\hline & 64,1 & 63,1 & 63,6 & 70,1 & 60,5 & 63,0 & 66,5 & 65,9 & 65,5 & 2 \\
\hline \multicolumn{11}{|l|}{ América del Sur } \\
\hline Bolivia (Estado Plurinacional de) & 25,0 & 27,8 & 29,7 & 34,8 & 34,8 & 37,4 & 42,5 & 44,8 & 46,8 & 87 \\
\hline Brasil & 44,9 & 51,8 & 59,7 & 67,3 & 65,7 & 79,8 & 87,9 & 77,7 & 74,5 & 66 \\
\hline Chile & 50,3 & 57,9 & 60,4 & 59,4 & 57,0 & 67,4 & 72,0 & 71,2 & 70,1 & 39 \\
\hline Colombia & 41,1 & 41,4 & 48,1 & 53,6 & 50,0 & 58,5 & 62,9 & 65,5 & 63,0 & 54 \\
\hline Ecuador & 39,4 & 41,3 & 42,9 & 47,9 & 47,9 & 50,8 & 52,6 & 54,3 & 55,1 & 40 \\
\hline Paraguay & 27,7 & 31,2 & 37,4 & 46,2 & 41,1 & 45,2 & 53,1 & 51,8 & 52,5 & 90 \\
\hline Perú & 40,9 & 43,1 & 44,5 & 47,2 & 46,3 & 51,5 & 55,2 & 57,7 & 55,9 & 37 \\
\hline Uruguay & 46,2 & 48,6 & 53,1 & 63,1 & 61,3 & 71,4 & 79,1 & 79,4 & 83,4 & 80 \\
\hline \multirow[t]{2}{*}{ Venezuela (República Bolivariana de) } & 40,8 & 45,4 & 51,1 & 65,2 & 69,8 & 50,9 & 63,3 & 70,9 & 69,7 & 71 \\
\hline & 43,7 & 49,1 & 55,5 & 62,6 & 61,4 & 70,0 & 77,3 & 72,4 & 70,0 & 60 \\
\hline América Latina y el Caribe & 48,2 & 52,2 & 56,7 & 61,9 & 58,4 & 65,7 & 71,5 & 67,5 & 66,5 & 38 \\
\hline
\end{tabular}

Fuente: Elaboración propia sobre la base de Comisión Económica para América Latina y el Caribe (CEPAL), Bases de Datos y Publicaciones Estadísticas (CEPALSTAT) [en línea] http://estadisticas.cepal.org/, y Base de Datos del Banco Mundial [en línea] http://data.worldbank.org/. 


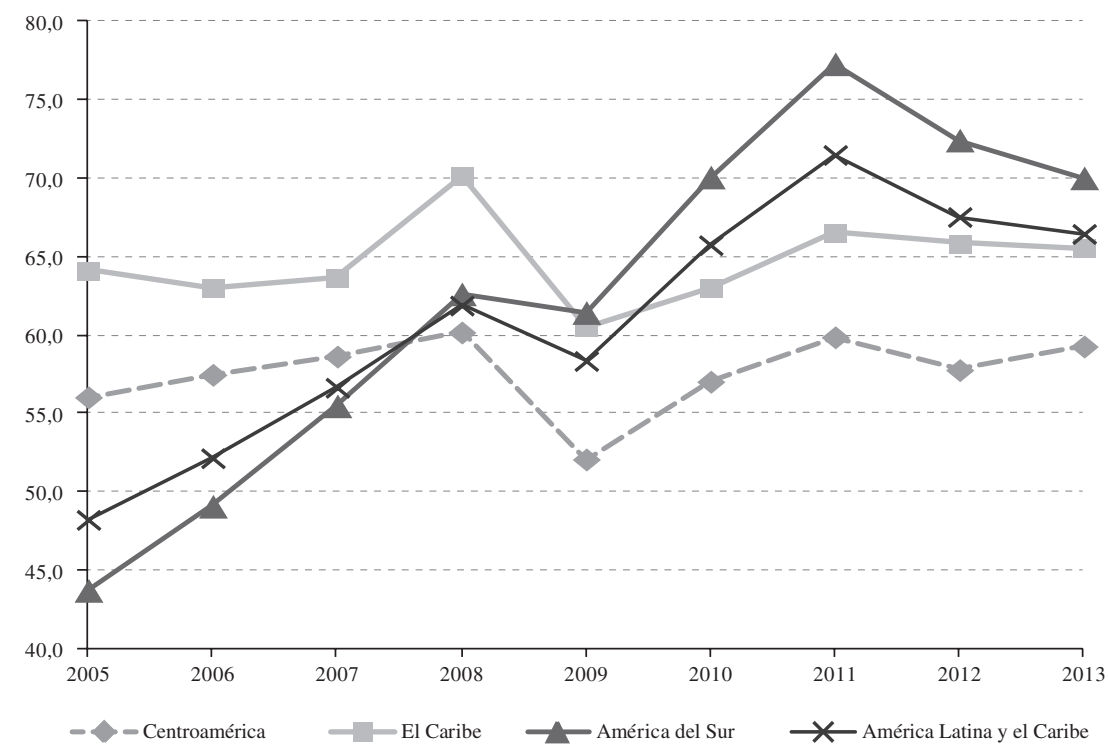

Fuente: Elaboración propia sobre la base de Comisión Económica para América Latina y el Caribe (CEPAL), Bases de Datos y Publicaciones Estadísticas (CEPALSTAT) [en línea] http://estadisticas.cepal.org/, y Base de Datos del Banco Mundial [en línea] http://data.worldbank.org/.

Las diferencias entre las regiones obedecen a dos factores principales: i) los precios del Caribe (y de Centroamérica, en menor medida) se encuentran más "atados" a los de los Estados Unidos de América que los de América del Sur; y ii) el efecto tipo de cambio, pues los tipos de cambio del Caribe son mucho más estables que los de América Latina, aunque con algunas excepciones, como es el caso de Jamaica y Suriname o de los países latinoamericanos cuyas economías son dolarizadas (Ecuador, Panamá y El Salvador). El primer factor se aplica principalmente a las diferencias de tendencias (comparación horizontal o temporal), mientras que el segundo explica parcialmente ambos tipos de diferencias, tanto de tendencias como geográficas (o verticales).

\section{Comparación de los resultados a nivel agregado y por componente (series a precios corrientes)}

Como ya se mencionó, uno de los objetivos del ejercicio consiste en comparar los resultados obtenidos a nivel agregado del PIB o mediante su desagregación por componentes. El ejercicio por componentes se llevó a cabo para los nueve países de América del Sur incluidos en este estudio y México, pues son los países de la región que participaron en las rondas de 2005 y 2011 y, por lo tanto, permiten comparar las estimaciones con respecto al referente. El cuadro 2 permite comparar los resultados obtenidos mediante ambos métodos, es decir, los cálculos elaborados a nivel del PIB agregado con aquellos realizados a nivel de sus componentes.

Corresponde señalar que se registran diferencias incluso para el año base 2011 (columna en gris en el cuadro 2), que superan un $3 \%$. A priori, se podía esperar que esas PPA debían ser iguales; sin embargo, existen diferencias en las ponderaciones de los componentes de cuentas nacionales, tanto para los países latinoamericanos como para el país de referencia, con respecto a aquellas utilizadas en 2011 en el contexto del PCI, lo que explica parcialmente las disparidades detectadas. Asimismo, la agregación multilateral se realizó para la estimación por componentes utilizando solo los 11 países involucrados (los diez de América Latina más los Estados Unidos de América), mientras que en el contexto del PCI, esa operación se llevó a cabo incluyendo a los 16 países de América Latina participantes en el programa, excluidos Chile y México que participaron en la "región" ocDE ${ }^{13}$.

Como se aprecia en el cuadro 2, existen diferencias importantes que tienden a ampliarse en la cola de las

\footnotetext{
13 Para más información sobre estas metodologías, véase Banco Mundial (2013).
} 
Estimaciones de las PPA a nivel del PIB y por componente, en unidades monetarias de cada país por 1 dólar, 2005-2013

\begin{tabular}{|c|c|c|c|c|c|c|c|c|c|c|}
\hline País & Estimación & 2005 & 2006 & 2007 & 2008 & 2009 & 2010 & 2011 & 2012 & 2013 \\
\hline \multirow{3}{*}{$\begin{array}{l}\text { Bolivia (Estado } \\
\text { Plurinacional de) }\end{array}$} & Nivel PIB & 2,02 & 2,23 & 2,33 & 2,52 & 2,44 & 2,62 & 2,95 & 3,10 & 3,23 \\
\hline & Componentes & 2,22 & 2,40 & 2,54 & 2,65 & 2,68 & 2,78 & 3,05 & 3,16 & 3,34 \\
\hline & Diferencia porcentual & 10 & 7,6 & 8,9 & 5,1 & 9,7 & 5,9 & 3,4 & 1,9 & 3,2 \\
\hline \multirow[t]{2}{*}{ Brasil } & Nivel PIB & 1,09 & 1,13 & 1,16 & 1,23 & 1,31 & 1,40 & 1,47 & 1,52 & 1,61 \\
\hline & Componentes & 1,14 & 1,17 & 1,20 & 1,25 & 1,35 & 1,42 & 1,47 & 1,54 & 1,64 \\
\hline \multirow[t]{3}{*}{ Chile } & Nivel PIB & 281,66 & 307,21 & 315,56 & 310,23 & 319,72 & 343,75 & 348,02 & 346,33 & 347,14 \\
\hline & Componentes & 305,38 & 304,00 & 310,57 & 331,14 & 339,08 & 343,77 & 349,92 & 355,75 & 362,25 \\
\hline & Diferencia porcentual & 8,4 & $-1,0$ & $-1,6$ & 6,7 & 6,1 & 0,0 & 0,5 & 2,7 & 4,4 \\
\hline \multirow[t]{3}{*}{ Colombia } & Nivel PIB & 952,72 & 977,70 & 1000,36 & 1055,24 & 1082,98 & 1111,17 & 1161,91 & 1177,60 & 1177,98 \\
\hline & Componentes & 1016,56 & 1038,43 & 1056,63 & 1085,38 & 1139,19 & 1148,59 & 1176,76 & 1192,26 & 1203,58 \\
\hline & Diferencia porcentual & 6,7 & 6,2 & 5,6 & 2,9 & 5,2 & 3,4 & 1,3 & 1,2 & 2,2 \\
\hline \multirow[t]{3}{*}{ Ecuador } & Nivel PIB & 0,39 & 0,41 & 0,43 & 0,48 & 0,48 & 0,51 & 0,53 & 0,54 & 0,55 \\
\hline & Componentes & 0,42 & 0,44 & 0,44 & 0,47 & 0,50 & 0,52 & 0,53 & 0,55 & 0,56 \\
\hline & Diferencia porcentual & 7,3 & 5,4 & 3,1 & $-0,9$ & 4,5 & 2,6 & 1,7 & 2,1 & 2,1 \\
\hline \multirow[t]{3}{*}{ México } & Nivel PIB & 6,40 & 6,60 & 6,75 & 7,02 & 7,20 & 7,43 & 7,67 & 7,78 & 7,79 \\
\hline & Componentes & 6,59 & 6,69 & 6,88 & 7,14 & 7,52 & 7,75 & 7,91 & 8,11 & 8,20 \\
\hline & Diferencia porcentual & 3,0 & 1,4 & 1,9 & 1,7 & 4,4 & 4,2 & 3,0 & 4,2 & 5,3 \\
\hline \multirow[t]{3}{*}{ Paraguay } & Nivel PIB & 1708,76 & 1758,67 & 1880,39 & 2016,39 & 2042,07 & 2140,51 & 2227,34 & 2291,93 & 2268,09 \\
\hline & Componentes & 1753,20 & 1866,89 & 1952,31 & 2100,37 & 2073,83 & 2210,40 & 2274,37 & 2281,62 & 2360,90 \\
\hline & Diferencia porcentual & 2,6 & 6,2 & 3,8 & 4,2 & 1,6 & 3,3 & 2,1 & $-0,4$ & 4,1 \\
\hline \multirow[t]{3}{*}{ Perú } & Nivel PIB & 1,35 & 1,41 & 1,39 & 1,38 & 1,40 & 1,46 & 1,52 & 1,52 & 1,51 \\
\hline & Componentes & 1,49 & 1,48 & 1,47 & 1,49 & 1,52 & 1,52 & 1,55 & 1,57 & 1,60 \\
\hline & Diferencia porcentual & 10,1 & 4,8 & 5,4 & 7,6 & 8,8 & 4,5 & 1,8 & 3,0 & 5,7 \\
\hline Uruguay & Diferencia porcentual & 4,3 & 5,1 & 4,9 & 2,5 & 4,1 & 2,4 & 0,3 & 0,4 & 0,1 \\
\hline \multirow{3}{*}{$\begin{array}{l}\text { Venezuela } \\
\text { (República } \\
\text { Bolivariana de) }\end{array}$} & Nivel PIB & 0,85 & 0,98 & 1,10 & 1,40 & 1,50 & 2,16 & 2,71 & 3,04 & 4,21 \\
\hline & Componentes & 0,89 & 0,98 & 1,11 & 1,35 & 1,74 & 2,18 & 2,67 & 3,10 & 4,70 \\
\hline & Diferencia porcentual & 3,8 & 0,6 & 1,0 & $-3,6$ & 16,1 & 0,9 & $-1,6$ & 2,0 & 11,5 \\
\hline
\end{tabular}

Fuente: Elaboración propia sobre la base de Comisión Económica para América Latina y el Caribe (CEPAL), Bases de Datos y Publicaciones Estadísticas (CEPALSTAT) [en línea] http://estadisticas.cepal.org/; y Base de Datos del Banco Mundial [en línea] http://data.worldbank.org/ Nota: PPA: Paridades del poder adquisitivo; PIB: Producto interno bruto.

series. Ese es el caso del Brasil, Colombia, el Ecuador, el Perú y el Uruguay, países en los que las PPA son similares en 2011, mientras que las diferencias se van acentuando al alejarse de ese año.

Cabe señalar que las PPA estimadas por componentes muestran valores generalmente mayores que las estimaciones a nivel del PIB. Por su parte, Venezuela (República Bolivariana de) y Chile presentan diferencias más irregulares debidas a cambios en los precios de los principales productos básicos que exportan (petróleo y cobre, respectivamente ${ }^{14}$ ). Esto se debe sobre todo a que en el método por componente se utiliza como PPA de referencia el tipo de cambio para las exportaciones e importaciones. El criterio detrás de esta práctica es que los precios de los productos básicos —bajo el

\footnotetext{
${ }^{14}$ En 2008 se registra una pronunciada caída en el precio del petróleo, lo que afectó no solo a Venezuela (República Bolivariana de) sino también al Ecuador (véase el gráfico 8); a su vez, el precio del cobre sufrió importantes descensos en 2005 y 2008-2009.
} 
marcado supuesto de ausencia de costos de transporte, seguros e impuestos, entre otros- deberían ser los mismos en dólares para todos los países, de tal forma que los precios relativos (y las PPA) deberían estar dados por el tipo de cambio ${ }^{15}$. Al extrapolar las PPA con el deflactor del PIB agregado, se está incorporando el efecto de las modificaciones de los precios en las canastas de exportación e importación de los países (pues intervienen en el cálculo del deflactor del PIB), lo que no se ve reflejado necesariamente en los tipos de cambio utilizados en la estimación por componentes.

En el gráfico 8 se reportan las series de PIB per cápita en PPA corrientes, cuyas paridades fueron estimadas a nivel agregado y por componentes.

${ }^{15}$ La situación de los servicios (por ejemplo, turismo) es diferente y mucho más compleja; por motivos de simplicidad, en el PCı se utilizan las tasas de cambio como PPA de referencia.
En general, los valores del PIB per cápita en PPA estimados por componentes son menores que aquellos estimados a nivel agregado. Estas diferencias pueden ser importantes para algunos países y llegar a valores cercanos al 10\% del PIB per cápita total en algunos años, como es el caso de Bolivia (Estado Plurinacional de).

Por las razones expuestas, Venezuela (República Bolivariana de) y Chile, y en menor medida el Ecuador, presentan características distintas, con diferencias en el PIB per cápita que pueden cambiar de signo en función del método utilizado. Por ejemplo, en Venezuela (República Bolivariana de), el método por componentes arroja un PIB per cápita en PPA de casi 650 dólares menor en 2008, mientras que al año siguiente el método agregado presenta un valor superior en casi 2.300 dólares.

Por otra parte, el PIB per cápita en PPA calculado mediante el método por componentes muestra un efecto más pronunciado de la crisis de 2009. Esto se puede apreciar en el gráfico 8 respecto de todos los países analizados, con excepción del Paraguay.

GRÁFICO 8

PIB per cápita en PPA estimado por el método agregado y por componentes, en dólares, 2005-2013
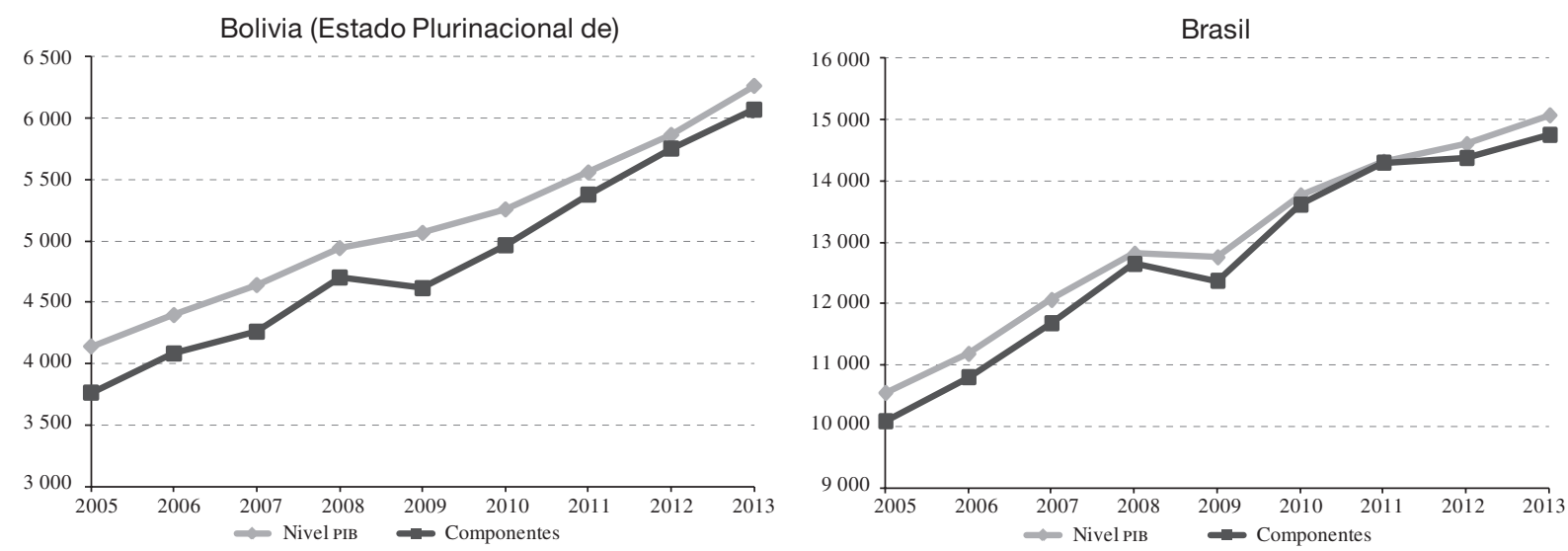

Chile
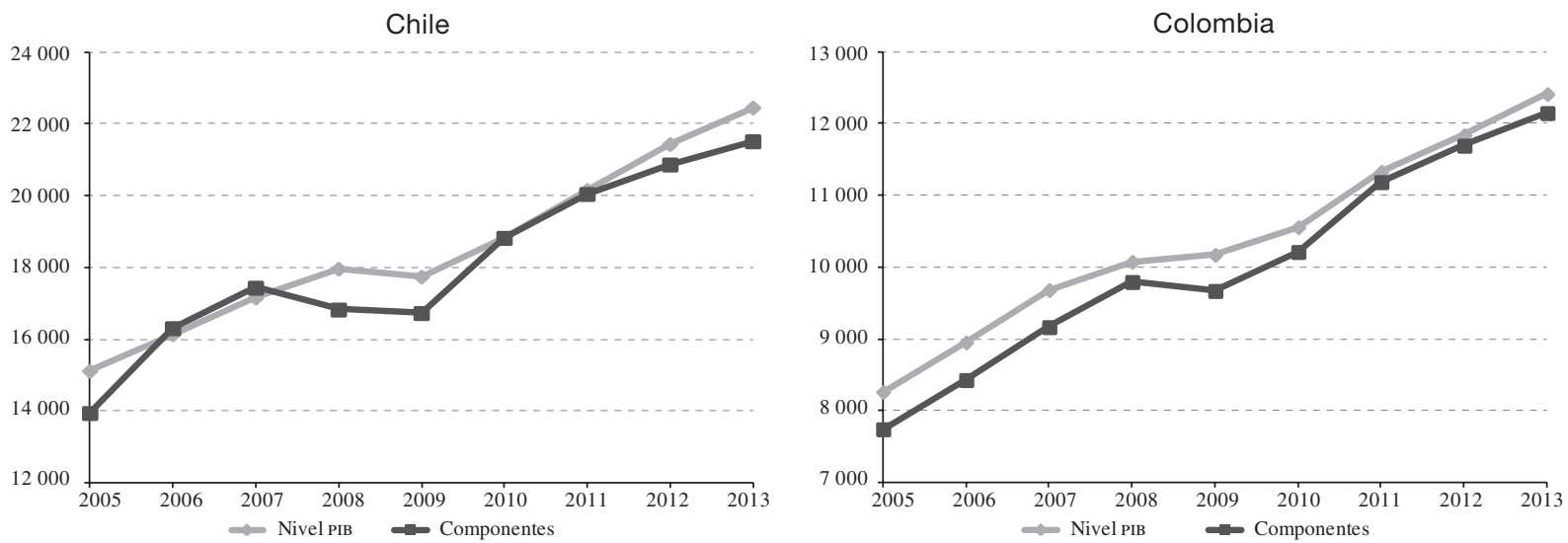
Gráfico 8 (conclusión)

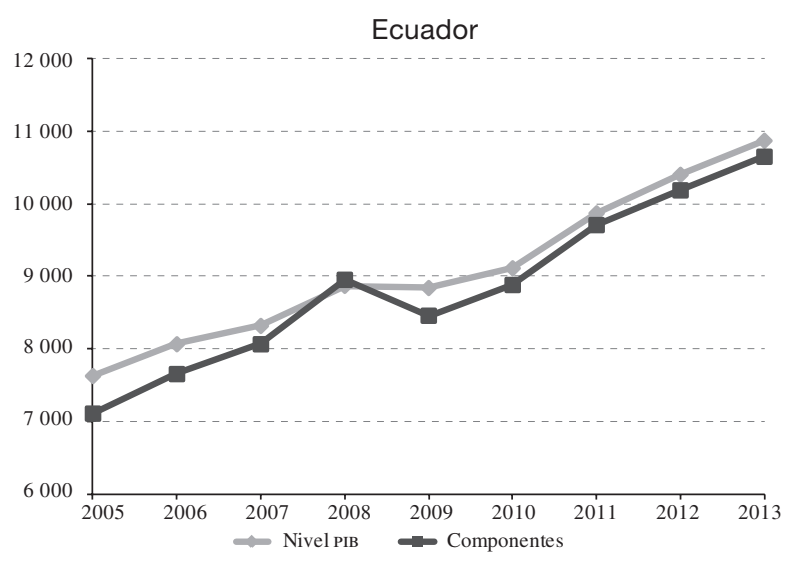

Paraguay

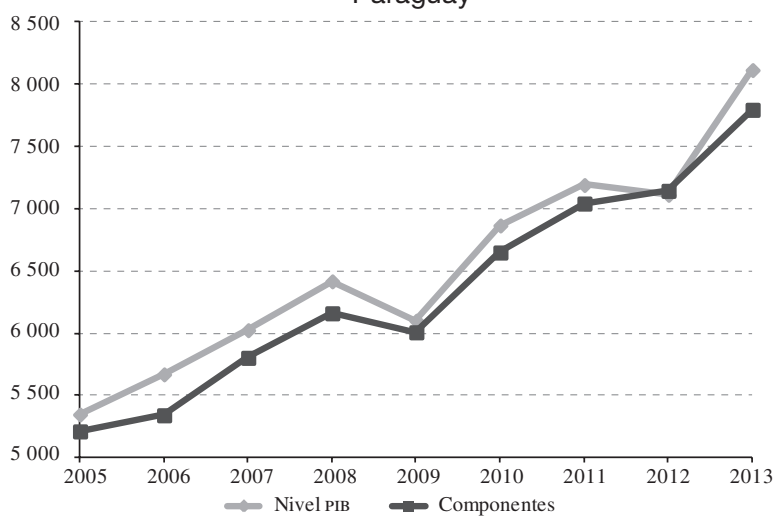

Uruguay

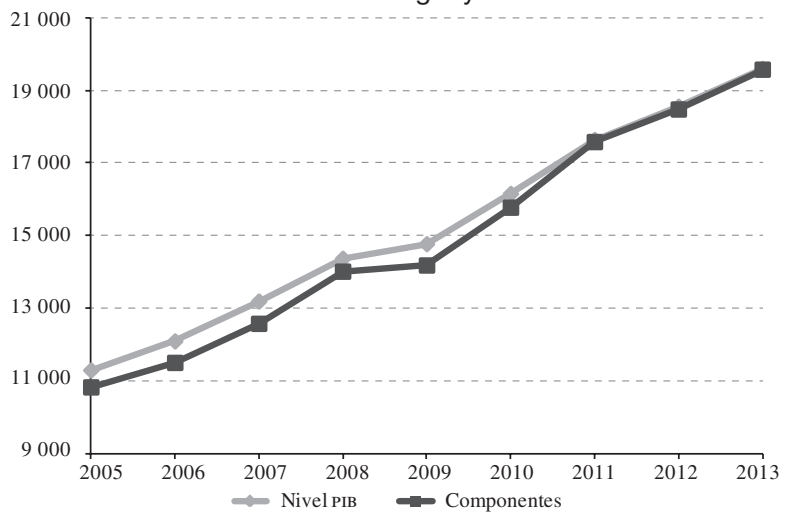

México

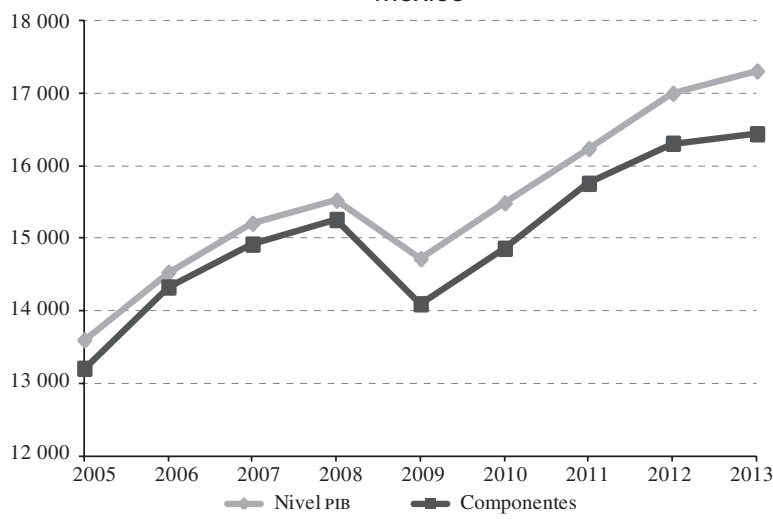

Perú

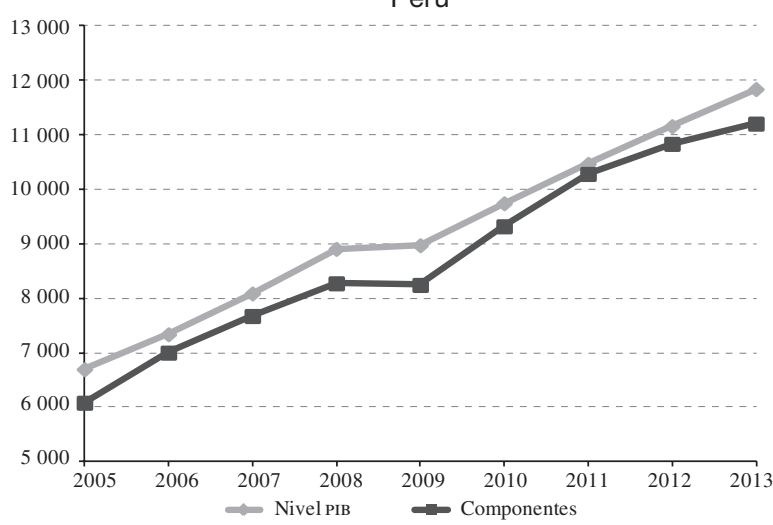

Venezuela (República Bolivariana de)

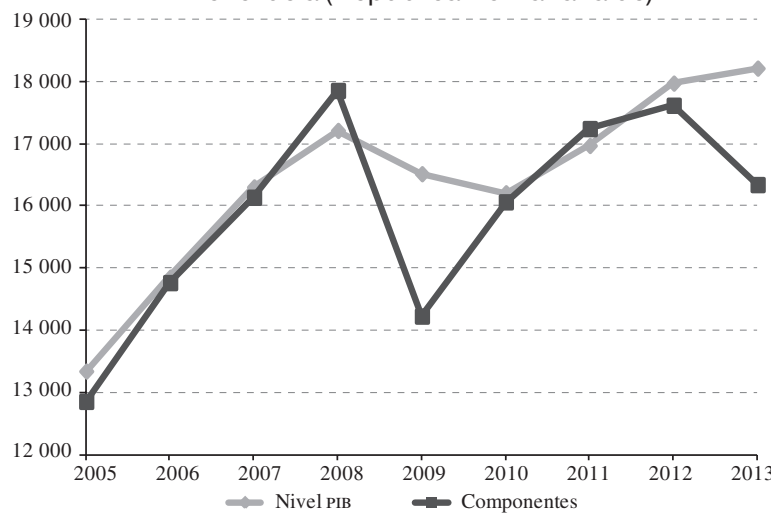

Fuente: Elaboración propia sobre la base de Comisión Económica para América Latina y el Caribe (CEPAL), Bases de Datos y Publicaciones Estadísticas (CEPALSTAT) [en línea] http://estadisticas.cepal.org/, y Base de Datos del Banco Mundial [en línea] http://data.worldbank.org/. Nota: PPA: Paridades del poder adquisitivo; PIB: Producto interno bruto. 
Finalmente, es importante mencionar que dada la naturaleza no aditiva del método de agregación EKS utilizado por el PCI y en este ejercicio, se crea una discrepancia estadística entre el PIB total real a PPA y la suma de los componentes a PPA, lo que dificulta el cálculo de la participación real de los componentes en el PIB y la estimación de agregados específicos como es el caso de la absorción doméstica. La magnitud de esta discrepancia varía notablemente entre los países, con valores que no superan el $1 \%$ para Colombia durante todo el período, y discrepancias que alcanzan casi el $15 \%$ del PIB total en Venezuela (República Bolivariana de) para 2005.

\section{Una comparación crucial: las estimaciones versus la referencia}

La comparación entre los resultados obtenidos a nivel agregado y los guarismos calculados por componentes no permite determinar cuál de las dos estimaciones es más "cercana a la realidad", aunque desde el punto de vista teórico se debería utilizar el método de los componentes, tal como lo recomienda el SCN 2008.

Una comparación "crucial" para verificar la bondad de los métodos de estimación radica en analizar los resultados obtenidos comparándolos con las estimaciones obtenidas en el marco de una ronda del PCI (referencia). Tratándose de un cálculo efectuado a partir de la comparación de precios relevados a nivel de productos elementales, con información abundante y detallada, es lógico asumir que esos resultados son de mejor calidad que cualquier extrapolación realizada a partir de los deflactores. En esta subsección se comparan los resultados de la ronda de 2005 del PCI con las PPA obtenidas mediante los dos métodos de extrapolación para ese año. En el cuadro 3 se presentan estos resultados.

Como puede apreciarse, las PPA por componentes presentan valores más cercanos al año de referencia 2005 para todos los países. Las PPA por componentes de algunos países, como es el caso de Bolivia (Estado Plurinacional de), el Ecuador y el Perú, muestran valores muy cercanos al período de referencia, con diferencias menores del $1 \%$, mientras que aquellas estimadas a nivel agregado del PIB presentan diferencias importantes de entre el 6\% y el 10\% para esos mismos países. Por su parte, en el caso del Brasil, el Paraguay, el Uruguay y Venezuela (República Bolivariana de), las PPA estimadas difieren notablemente de aquellas obtenidas en la ronda de 2005, diferencias que ponen de manifiesto la debilidad que tienen los métodos de extrapolación/retropolación.

Por otra parte, es importante tener en cuenta que los resultados del PCI correspondientes al año 2005 son también estimaciones y, como tales, pueden incorporar sesgos. En este sentido, Deaton y Atten (2014) afirman que las PPA calculadas en el contexto de la ronda de 2005 del PCI, en especial aquellas referentes al consumo de los hogares, se encuentran sobrestimadas debido principalmente a la metodología utilizada para vincular las diferentes regiones. Según los autores citados, esta sobrestimación es mayor para las regiones de Asia, Asia occidental y África, aunque también afecta a los países de América Latina. De ser así, las PPA "verdaderas" se encontrarían más cercanas a las extrapolaciones realizadas en este estudio.

CUADRO 3

Comparación de las PPA calculadas en la ronda 2005 del PCI con las estimaciones obtenidas mediante los métodos de extra/retropolación, 2005

\begin{tabular}{|c|c|c|c|c|c|}
\hline País & PPA PCI 2005 & Nivel PIB & Componentes & $\begin{array}{l}\text { Diferencia nivel } \\
\text { PIB }\end{array}$ & $\begin{array}{c}\text { Diferencia } \\
\text { componentes }\end{array}$ \\
\hline Bolivia (Estado Plurinacional de) & 2,23 & 2,02 & 2,22 & $-9,4 \%$ & $-0,4 \%$ \\
\hline Brasil & 1,36 & 1,09 & 1,14 & $-19,6 \%$ & $-15,9 \%$ \\
\hline Chile & 333,69 & 281,66 & 305,38 & $-15,6 \%$ & $-8,5 \%$ \\
\hline Colombia & 1081,95 & 952,72 & 1016,56 & $-11,9 \%$ & $-6,0 \%$ \\
\hline Ecuador & 0,42 & 0,39 & 0,42 & $-6,1 \%$ & $0,7 \%$ \\
\hline México & 7,13 & 6,40 & 6,59 & $-10,3 \%$ & $-7,6 \%$ \\
\hline Paraguay & 2006,83 & 1708,76 & 1753,20 & $-14,9 \%$ & $-12,6 \%$ \\
\hline Perú & 1,49 & 1,35 & 1,49 & $-9,4 \%$ & $-0,3 \%$ \\
\hline Uruguay & 13,28 & 11,32 & 11,81 & $-14,8 \%$ & $-11,1 \%$ \\
\hline Venezuela (República Bolivariana de) ${ }^{\mathrm{a}}$ & 1,15 & 0,85 & 0,89 & $-26,0 \%$ & $-23,2 \%$ \\
\hline
\end{tabular}

Fuente: Elaboración propia sobre la base de Comisión Económica para América Latina y el Caribe (CEPAL), Bases de Datos y Publicaciones Estadísticas (CEPALSTAT) [en línea] http://estadisticas.cepal.org/, y Base de Datos del Banco Mundial [en línea] http://data.worldbank.org/.

Nota: PPA: Paridades del poder adquisitivo; PCI: Programa de Comparación Internacional.

a Las PPa de Venezuela (Rep. Bol. de) para el PCI de 2005 fueron divididas por 1.000 debido al cambio de moneda implementado en 2008 , cuando se pasa del bolívar al bolívar fuerte, a una tasa de 1.000 a 1. 


\section{IV}

\section{Consideraciones finales}

En el presente trabajo se investigan distintas opciones para obtener series de PPA, sin incurrir en mayores costos adicionales.

Existen varias limitaciones en lo que se refiere a la disponibilidad de información, sobre todo para aquellos países que no participaron directamente en las rondas de 2005 o 2011 del Programa de Comparación Internacional (PCI); también existen limitaciones de índole metodológica, pues a pesar de haber utilizado las recomendaciones del SCN 2008 para estimar las series de PPA, los resultados pueden incorporar sesgos importantes derivados de un "efecto estructura". Estos sesgos pueden ser reducidos al realizar la extrapolación a niveles más desagregados, como es el caso del método de componentes. Sin embargo, la implementación de este método no es viable para los países del Caribe, pues la mayoría de países de esta subregión no compilan el PIB por tipo de gasto a precios constantes y, por lo tanto, no se dispone de los deflactores por tipo de gasto.

Por consiguiente, para mejorar los resultados y mitigar los sesgos de estimación, es necesario realizar los cálculos por componente, descendiendo - aun más-en el caso del consumo de los hogares, componente que amerita un análisis especial por su elevada participación en el PIB.

Asimismo, en este artículo se han señalado otros factores que pueden introducir discrepancias entre las estimaciones y los valores obtenidos en los años de referencia. Una primera diferencia radica en el hecho de que los cálculos de las PPA se realizan de forma multilateral, lo que implica que un cambio en la información de uno de los países involucrados afecta a las estimaciones de todas las PPA de los países. En este sentido, si el conjunto de países participantes en el cálculo no es el mismo, puede haber efectos importantes en los resultados finales.

En el caso del método por componentes, en los resultados se incorporan a solo diez países de América Latina y a los Estados Unidos de América como referencia, por razones de comparabilidad y disponibilidad de la información para las rondas del PCI de 2005 y 2011.

La segunda diferencia está dada por cambios metodológicos implementados entre las rondas de 2005 y 2011 del PCI, en cuyo contexto se realizan, en una primera etapa, cálculos de las PPA a niveles regionales; luego, las regiones son vinculadas (linked) por medio de distintas metodologías. La metodología utilizada para vincular los resultados regionales cambió significativamente entre ambas rondas: en 2005 se empleó una metodología de anillos (rings), en la que solo participaron del cálculo global 18 países, representando a todas las regiones participantes. En cambio, en 2011 se utilizó una canasta común global, usando información de todos los países participantes.

Según Deaton y Aten (2014), esta diferencia metodológica es responsable de gran parte de la sobrestimación generalizada de las PPA del consumo de los hogares (y, por ende, del PIB) en la ronda 2005. Según los autores, esta sobrestimación obedece sobre todo a dos factores: i) la inclusión de productos en la "canasta de anillos" que se encuentran disponibles solo en países desarrollados y que, por lo tanto, son caros en países más pobres, y ii) el uso de ponderaciones promedio entre países en la metodología de anillos, la que tiene por efecto que algunos productos caros, pero con poco peso en el gasto de un país en desarrollo (como el transporte aéreo), tengan mayor peso en el cálculo por anillos.

A pesar de las restricciones mencionadas, las series elaboradas para este trabajo respetan los estándares y recomendaciones internacionales y pueden ser utilizadas para realizar estudios comparativos entre los países. Desde una perspectiva analítica, esos resultados constituyen un importante insumo para probar la hipótesis de carácter interpretativo sobre los diferenciales en el nivel y evolución de los precios en los países de la región; entre ellas, las brechas existentes en el grado de apertura comercial y arancelaria o en las productividades entre los países de la región, o, aun más, en las características de sus matrices productivas y de sus mercados. 
ANEXO

CUADRO A.1

América Latina y el Caribe: paridades de poder adquisitivo (PPA) ${ }^{\mathrm{a}}$, 2005-2013

(En unidades de moneda nacional por dólar)

\begin{tabular}{|c|c|c|c|c|c|c|c|c|c|}
\hline País & 2005 & 2006 & 2007 & 2008 & 2009 & 2010 & 2011 & 2012 & 2013 \\
\hline Antigua y Barbuda & 1,71 & 1,67 & 1,68 & 1,72 & 1,74 & 1,74 & 1,73 & 1,74 & 1,71 \\
\hline Bahamas & 1,03 & 1,01 & 1,01 & 1,01 & 0,99 & 0,98 & 0,95 & 0,96 & 0,96 \\
\hline Barbados & 2,11 & 2,14 & 2,15 & 2,11 & 2,21 & 2,10 & 2,02 & 1,91 & 1,93 \\
\hline Belice & 1,12 & 1,14 & 1,16 & 1,17 & 1,13 & 1,13 & 1,15 & 1,15 & 1,15 \\
\hline Bolivia (Estado Plurinacional de) & 2,02 & 2,23 & 2,33 & 2,52 & 2,44 & 2,62 & 2,95 & 3,10 & 3,23 \\
\hline Brasil & 1,09 & 1,13 & 1,16 & 1,23 & 1,31 & 1,40 & 1,47 & 1,52 & 1,61 \\
\hline Chile & 281,66 & 307,21 & 315,56 & 310,23 & 319,72 & 343,75 & 348,02 & 346,33 & 347,14 \\
\hline Colombia & 952,72 & 977,70 & 1000,36 & 1055,24 & 1082,98 & 1111,17 & 1161,91 & 1177,60 & 1177,98 \\
\hline Costa Rica & 233,30 & 251,24 & 267,69 & 295,09 & 317,41 & 338,54 & 346,74 & 354,55 & 366,83 \\
\hline Dominica & 1,82 & 1,81 & 1,79 & 1,79 & 1,91 & 1,84 & 1,86 & 1,88 & 1,87 \\
\hline Ecuador & 0,39 & 0,41 & 0,43 & 0,48 & 0,48 & 0,51 & 0,53 & 0,54 & 0,55 \\
\hline El Salvador & 0,46 & 0,46 & 0,47 & 0,49 & 0,48 & 0,49 & 0,50 & 0,50 & 0,49 \\
\hline Granada & 1,72 & 1,75 & 1,74 & 1,84 & 1,83 & 1,82 & 1,78 & 1,82 & 1,83 \\
\hline Guatemala & 2,84 & 2,89 & 3,02 & 3,24 & 3,33 & 3,46 & 3,63 & 3,68 & 3,75 \\
\hline Guyana & 83,85 & 85,93 & 94,34 & 100,87 & 102,24 & 107,78 & 114,43 & 118,87 & 117,33 \\
\hline Haití & 13,04 & 14,52 & 15,28 & 16,92 & 17,41 & 18,14 & 19,11 & 19,76 & 20,76 \\
\hline Honduras & 7,57 & 7,74 & 8,04 & 8,50 & 9,08 & 9,39 & 9,91 & 10,09 & 10,09 \\
\hline Jamaica & 39,26 & 41,48 & 38,96 & 43,35 & 48,10 & 52,24 & 54,12 & 56,69 & 60,38 \\
\hline México & 6,40 & 6,60 & 6,75 & 7,02 & 7,20 & 7,43 & 7,67 & 7,78 & 7,79 \\
\hline Nicaragua & 5,72 & 6,00 & 6,41 & 7,30 & 7,73 & 8,27 & 8,92 & 9,43 & 9,86 \\
\hline Panamá & 0,49 & 0,49 & 0,49 & 0,52 & 0,52 & 0,54 & 0,55 & 0,56 & 0,57 \\
\hline Paraguay & 1708,76 & 1758,67 & 1880,39 & 2016,39 & 2042,07 & 2140,51 & 2227,34 & 2291,93 & 2268,09 \\
\hline Perú & 1,35 & 1,41 & 1,39 & 1,38 & 1,40 & 1,46 & 1,52 & 1,52 & 1,51 \\
\hline República Dominicana & 14,90 & 15,23 & 15,68 & 16,98 & 17,43 & 18,19 & 19,45 & 19,86 & 20,17 \\
\hline Saint Kitts y Nevis & 1,59 & 1,71 & 1,74 & 1,76 & 1,78 & 1,78 & 1,80 & 1,80 & 1,78 \\
\hline San Vicente y las Granadinas & 1,65 & 1,65 & 1,74 & 1,70 & 1,68 & 1,73 & 1,69 & 1,68 & 1,67 \\
\hline Santa Lucía & 1,69 & 1,73 & 1,81 & 1,76 & 1,74 & 1,84 & 1,84 & 1,86 & 1,89 \\
\hline Suriname & 1,21 & 1,24 & 1,28 & 1,45 & 1,54 & 1,63 & 1,83 & 1,99 & 2,02 \\
\hline Trinidad y Tabago & 3,40 & 3,32 & 3,66 & 4,44 & 3,19 & 3,40 & 3,94 & 3,78 & 3,86 \\
\hline Uruguay & 11,32 & 11,70 & 12,47 & 13,21 & 13,84 & 14,31 & 15,28 & 16,12 & 17,09 \\
\hline Venezuela (República Bolivariana de) & 0,85 & 0,98 & 1,10 & 1,40 & 1,50 & 2,16 & 2,71 & 3,04 & 4,21 \\
\hline
\end{tabular}

Fuente: Elaboración propia sobre la base de Comisión Económica para América Latina y el Caribe (CEPAL), Bases de Datos y Publicaciones Estadísticas (CEPALSTAT) [en línea] http://estadisticas.cepal.org/, y Base de Datos del Banco Mundial [en línea] http://data.worldbank.org/.

a Estimadas a través del método agregado. 
CUADRO A.2

América Latina y el Caribe: PIB total en PPA, a precios corrientesa ${ }^{a}$ 2005-2013 (En millones de dólares)

\begin{tabular}{|c|c|c|c|c|c|c|c|c|c|}
\hline Región o país & 2005 & 2006 & 2007 & 2008 & 2009 & 2010 & 2011 & 2012 & 2013 \\
\hline Antigua y Barbuda & 1574 & 1840 & 2068 & 2110 & 1870 & 1758 & 1762 & 1866 & 1892 \\
\hline Bahamas & 7448 & 7870 & 8196 & 8163 & 7881 & 8100 & 8356 & 8593 & 8780 \\
\hline Barbados & 3694 & 4025 & 4201 & 4298 & 4152 & 4213 & 4333 & 4421 & 4500 \\
\hline Belice & 1986 & 2141 & 2222 & 2339 & 2373 & 2482 & 2587 & 2734 & 2817 \\
\hline Bolivia (Estado Plurinacional de) & 38135 & 41192 & 44219 & 47858 & 49840 & 52531 & 56390 & 60373 & 65424 \\
\hline Brasil & 1963425 & 2103824 & 2291370 & 2457133 & 2467637 & 2685941 & 2816317 & 2896444 & 3012883 \\
\hline Colombia & 357038 & 392654 & 430919 & 454954 & 465981 & 490405 & 533513 & 565082 & 600331 \\
\hline Costa Rica & 40888 & 45844 & 50799 & 53210 & 53069 & 56379 & 60138 & 64361 & 67604 \\
\hline Dominica & 550 & 595 & 648 & 710 & 707 & 723 & 738 & 740 & 745 \\
\hline Ecuador & 105272 & 113284 & 118845 & 128879 & 130594 & 136849 & 150664 & 161376 & 171382 \\
\hline El Salvador & 37343 & 39996 & 42637 & 44028 & 42972 & 44090 & 45998 & 47705 & 49227 \\
\hline Granada & 1090 & 1079 & 1175 & 1210 & 1138 & 1146 & 1179 & 1186 & 1233 \\
\hline Guatemala & 73103 & 79402 & 86654 & 91252 & 92429 & 96242 & 102318 & 107248 & 112852 \\
\hline Haití & 12883 & 13578 & 14405 & 14812 & 15384 & 14716 & 15849 & 16599 & 17571 \\
\hline Jamaica & 17831 & 18907 & 22727 & 23007 & 22153 & 22092 & 22931 & 23211 & 23689 \\
\hline México & 1473580 & 1594433 & 1689647 & 1746559 & 1676347 & 1785035 & 1893303 & 2004931 & 2064130 \\
\hline Nicaragua & 18506 & 19867 & 21474 & 22520 & 22065 & 23072 & 24888 & 26590 & 28229 \\
\hline Panamá & 33434 & 37400 & 43046 & 47904 & 50187 & 53772 & 60793 & 68226 & 75026 \\
\hline Paraguay & 31580 & 34115 & 36921 & 40039 & 38744 & 44351 & 47233 & 47485 & 55048 \\
\hline Perú & 185811 & 205939 & 229428 & 255316 & 259955 & 285366 & 310051 & 334398 & 358920 \\
\hline República Dominicana & 73048 & 83327 & 92794 & 97588 & 99250 & 108802 & 114299 & 119445 & 126782 \\
\hline Saint Kitts y Nevis & 922 & 1006 & 1062 & 1127 & 1072 & 1050 & 1090 & 1096 & 1159 \\
\hline San Vicente y las Granadinas & 903 & 1002 & 1063 & 1102 & 1087 & 1063 & 1080 & 1112 & 1147 \\
\hline Santa Lucía & 1496 & 1669 & 1705 & 1799 & 1825 & 1828 & 1889 & 1892 & 1912 \\
\hline Suriname & 5071 & 5820 & 6280 & 6669 & 6922 & 7368 & 7916 & 8300 & 8667 \\
\hline Trinidad y Tabago & 29579 & 34881 & 37410 & 39437 & 37992 & 38537 & 38317 & 39491 & 40737 \\
\hline Uruguay & 37549 & 40289 & 44067 & 48156 & 49662 & 54493 & 59702 & 63008 & 66758 \\
\hline Venezuela (República Bolivariana de) & 356492 & 403718 & 450741 & 483837 & 471902 & 470552 & 500326 & 537963 & 553312 \\
\hline América Latina y el Caribe & 5181742 & 5622887 & 6091999 & 6459414 & 6407207 & 6847769 & 7266591 & 7625912 & 7955333 \\
\hline
\end{tabular}

Fuente: Elaboración propia sobre la base de Comisión Económica para América Latina y el Caribe (CEPAL), Bases de Datos y Publicaciones Estadísticas (CEPALSTAT) [en línea] http://estadisticas.cepal.org/, y Base de Datos del Banco Mundial [en línea] http://data.worldbank.org/.

Nota: PIB: Producto interno bruto; PPA: Paridades del poder adquisitivo.

a Estimadas mediante el método agregado. 
CUADRO A.3

América Latina y el Caribe: PIB total en PPA, a precios constantes ${ }^{a}, \mathbf{2 0 0 5 - 2 0 1 3}$

(En millones de dólares de 2010)

\begin{tabular}{|c|c|c|c|c|c|c|c|c|c|}
\hline Región o país & 2005 & 2006 & 2007 & 2008 & 2009 & 2010 & 2011 & 2012 & 2013 \\
\hline Antigua y Barbuda & 1732 & 1964 & 2151 & 2152 & 1893 & 1758 & 1726 & 1796 & 1794 \\
\hline Bahamas & 8195 & 8402 & 8523 & 8325 & 7978 & 8100 & 8187 & 8271 & 8326 \\
\hline Barbados & 4065 & 4297 & 4369 & 4384 & 4202 & 4213 & 4245 & 4255 & 4268 \\
\hline Belice & 2185 & 2285 & 2311 & 2385 & 2402 & 2482 & 2534 & 2631 & 2671 \\
\hline Bolivia (Estado Plurinacional de) & 41963 & 43976 & 45983 & 48810 & 50449 & 52531 & 55249 & 58109 & 62046 \\
\hline Brasil & 2160497 & 2245989 & 2382801 & 2506030 & 2497767 & 2685941 & 2759343 & 2787792 & 2857289 \\
\hline Chile & 272054 & 284541 & 297630 & 308531 & 305333 & 322929 & 341785 & 360179 & 374855 \\
\hline Colombia & 392874 & 419187 & 448114 & 464008 & 471671 & 490405 & 522720 & 543885 & 569328 \\
\hline Costa Rica & 44992 & 48942 & 52826 & 54269 & 53717 & 56379 & 58922 & 61947 & 64113 \\
\hline Dominica & 605 & 635 & 674 & 724 & 716 & 723 & 723 & 713 & 706 \\
\hline Ecuador & 115838 & 120939 & 123587 & 131444 & 132189 & 136849 & 147616 & 155322 & 162532 \\
\hline El Salvador & 41092 & 42699 & 44339 & 44904 & 43497 & 44090 & 45068 & 45916 & 46685 \\
\hline Granada & 1200 & 1152 & 1222 & 1234 & 1152 & 1146 & 1155 & 1142 & 1169 \\
\hline Guatemala & 80440 & 84768 & 90111 & 93068 & 93558 & 96242 & 100248 & 103225 & 107024 \\
\hline Haití & 14176 & 14495 & 14980 & 15106 & 15572 & 14716 & 15529 & 15977 & 16663 \\
\hline Honduras & 26709 & 28463 & 30225 & 31504 & 30738 & 31885 & 33108 & 34475 & 35381 \\
\hline Jamaica & 19621 & 20184 & 23634 & 23465 & 22424 & 22092 & 22467 & 22340 & 22465 \\
\hline México & 1621486 & 1702176 & 1757069 & 1781315 & 1696815 & 1785035 & 1855001 & 1929722 & 1957533 \\
\hline Nicaragua & 20364 & 21209 & 22331 & 22968 & 22334 & 23072 & 24385 & 25593 & 26771 \\
\hline Panamá & 36790 & 39927 & 44763 & 48858 & 50799 & 53772 & 59564 & 65667 & 71152 \\
\hline Paraguay & 34749 & 36420 & 38394 & 40836 & 39217 & 44351 & 46277 & 45704 & 52205 \\
\hline Perú & 204461 & 219855 & 238583 & 260397 & 263129 & 285366 & 303778 & 321854 & 340384 \\
\hline República Dominicana & 80380 & 88958 & 96497 & 99530 & 100462 & 108802 & 111987 & 114964 & 120235 \\
\hline Saint Kitts y Nevis & 1014 & 1074 & 1104 & 1150 & 1085 & 1050 & 1068 & 1055 & 1100 \\
\hline San Vicente y las Granadinas & 994 & 1070 & 1106 & 1124 & 1100 & 1063 & 1058 & 1070 & 1088 \\
\hline Santa Lucía & 1646 & 1781 & 1773 & 1835 & 1847 & 1828 & 1850 & 1821 & 1813 \\
\hline Suriname & 5580 & 6213 & 6531 & 6801 & 7006 & 7368 & 7756 & 7989 & 8219 \\
\hline Trinidad y Tabago & 32548 & 37238 & 38903 & 40222 & 38456 & 38537 & 37542 & 38009 & 38633 \\
\hline Uruguay & 41318 & 43012 & 45825 & 49114 & 50269 & 54493 & 58494 & 60644 & 63310 \\
\hline Venezuela (República Bolivariana de) & 392273 & 430999 & 468727 & 493466 & 477663 & 470552 & 490204 & 517783 & 524737 \\
\hline & 701842 & 6002851 & & 6587958 & & & 586 & & 7544497 \\
\hline América Latina & 5622457 & 5916555 & 6242786 & 6494157 & 6395179 & 6757409 & 7029275 & 7248756 & 7452243 \\
\hline El Caribe & 79385 & 86296 & 92299 & 93801 & 90261 & 90360 & 90311 & 91092 & 92254 \\
\hline
\end{tabular}

Fuente: Elaboración propia sobre la base de Comisión Económica para América Latina y el Caribe (CEPAL), Bases de Datos y Publicaciones Estadísticas (CEPALSTAT) [en línea] http://estadisticas.cepal.org/, y Base de Datos del Banco Mundial [en línea] http://data.worldbank.org/.

Nota: PIB: Producto interno bruto; PPA: Paridades del poder adquisitivo.

a Estimadas a través del método agregado. 


\section{Bibliografia}

Banco Mundial (2013), Measuring the Real Size of the Economy: The Framework, Methodology, and Results of the International Comparison Program (ICP), Washington, D.C.

(2008a), "Tables of results", 2005 International Comparison Program: Global Purchasing Power Parities and Real Expenditures, Washington, D.C.

(2008b), 2005 International Comparison Program (ICP): Global Purchasing Power Parities and Real Expenditures, Washington, D.C

(s/f), "Datos de libre acceso del Banco Mundial" [en línea] http://datos.bancomundial.org/frontpage.

(s/f), "2011 global results" [en línea] http://icp.worldbank. org/

CE/FMI/OCDE/Naciones Unidas/Banco Mundial (Comisión Europea/ Fondo Monetario Internacional/Organización para la Cooperación y el Desarrollo Económicos/Naciones Unidas/ Banco Mundial) (2009), Sistema de Cuentas Nacionales 2008, Nueva York.

CEPAL (Comisión Económica para América Latina y el Caribe) (2007), "Resultados del Programa de Comparación Internacional para América del Sur", Documentos de Proyectos (LC/W.138), Santiago.

CEPAlstat (Bases de Datos y Publicaciones Estadísticas) (s/f) [en línea] http://estadisticas.cepal.org.

Dalgaard, E. y H. Sorensen (2002), "Consistency between PPP benchmarks and national price and volume indices", documento preparado para la 27a Conferencia General de la Asociación Internacional de Investigaciones sobre Rentas y Riqueza, Statistics Denmark [en línea] www.oecd.org/std/ na/1957096.doc.

Deaton, A. y B. Aten (2014), "Trying to understand the PPPs in ICP 2011: why are the results so different?", NBER Working Paper, $\mathrm{N}^{\circ}$ 20244, Cambridge, Massachusetts, National Bureau of Economic Research.

Dykstra, S., C. Kenny y J. Sandefur (2014), "Global absolute poverty fell by almost half on Tuesday", Center for Global Development [en línea] http://www.cgdev.org/blog/globalabsolute-poverty-fell-almost-half-tuesday.

Epstein, H. y S. Marconi (2014), "América Latina y el Caribe: estimación de las series en paridades de poder adquisitivo (PPA). Un ejercicio preliminar para el período 2000-2011", serie Estudios Estadísticos, $\mathrm{N}^{\circ} 85$ (LC/L.3781), Santiago, Comisión Económica para América Latina y el Caribe (CEPAL).

FMI (Fondo Monetario Internacional), Estadísticas Financieras Internacionales [en línea] http://elibrary-data.imf.org/ QueryBuilder.aspx?key=19784651\&s=322.

Heston, A. (varios años), Penn World Tables (PWT) [en línea] https:// pwt.sas.upenn.edu/php_site/pwt_index.php.

Naciones Unidas (s/f), División de Estadística de las Naciones Unidas [en línea] http://unstats.un.org/unsd/mdg/ Metadata.aspx?Indic atorId $=0 \&$ SeriesId $=699$ 